\title{
Assessing the accuracy of land cover change with imperfect ground reference data
}

Foody, G. M.

Remote Sensing of Environment, 114, 2271-2285 (2010)

The manuscript of the above article revised after peer review and submitted to the journal for publication, follows. Please note that small changes may have been made after submission and the definitive version is that subsequently published as:

Foody, G. M., 2010. Assessing the accuracy of land cover change with imperfect ground reference data, Remote Sensing of Environment, 114, 2271-2285. 


\title{
Assessing the accuracy of land cover change with imperfect ground reference data
}

\author{
Giles M. Foody \\ School of Geography \\ University of Nottingham \\ Nottingham \\ NG7 2RD \\ UK
}

\begin{abstract}
The ground data used as a reference in the validation of land cover change products are often not an ideal gold standard but degraded by error. The effects of ground reference data error on the accuracy of land cover change detection and the accuracy of estimates of the extent of change was evaluated. Twelve data sets were simulated to allow the exploration of the impacts of a spectrum of ground data imperfections on the estimation of the producer's and user's accuracy of change as well as of change extent. Simulated data were used since this ensured that the actual properties of the data were known and to exclude effects due to other sources of ground reference data error; although the impacts of simulated reference data on two real confusion matrices is also illustrated. The imperfections evaluated ranged from the inclusion of small amounts of known error into the ground reference data through to the extreme situation in which ground data were absent. The results show that even small amounts of error in the ground reference data can introduce large error into studies of land cover change by remote sensing and reinforce the desire to avoid the expression ground truth as this might imply that the data are a gold standard reference. The effect of reference data imperfections was dependent on the degree of association between the errors in the cross tabulated data sets. For
\end{abstract}


example, in the scenarios investigated, a $10 \%$ error in the reference data set introduced an under-estimation of the producer's accuracy of $18.5 \%$ if the errors were independent but an over-estimation of the producer's accuracy of $12.3 \%$ if the errors were correlated. The magnitude of the mis-estimation of the producer's accuracy was also a function of the amount of change and greatest at low levels of change. The amount of land cover change estimated also varied greatly as a function of ground reference data error. Some possible methods to reduce or even remove the impacts of ground reference data error were illustrated. These ranged from simple algebraic means to estimate the actual values of accuracy and change extent if the imperfections were known through to a latent class analysis that allowed the assessment of classification accuracy and estimation of change extent without the use of ground reference data if the underlying model is defined appropriately.

\section{Introduction}

Land cover and land cover change are critical variables in major environmental issues of importance to the human-environmental sciences (Turner II et al., 2007). Land cover change is, for example, both a cause and a consequence of climate change. One major role of land cover change is as a source of enhanced atmospheric $\mathrm{CO}_{2}$ that contributes to global warming and which may in turn result in further land cover changes (Dale, 1997; McAlpine et al., 2009). Furthermore, land cover change has substantial impacts on a vast array of environmental systems including hydrological (Eshleman, 2004), ecological (Vitousek, 1994) and geomorphological (Foulds and Macklin, 2006). Land cover change 
is, for example, one of the greatest causes of biodiversity loss and hence a central variable in studies of biodiversity conservation (Duro et al., 2007; Gillespie et al., 2008; Jones et al., 2009). Accurate and up-to-date information on land cover and land cover change is, therefore, required for many applications.

Remote sensing is an attractive source of information on land cover and its dynamics at a range of spatial and temporal scales. However, numerous challenges are, or are perceived to be, encountered with the use of remote sensing for the derivation of information on land cover (Foody, 2002, 2008; Rindfuss et al., 2004; Strahler et al., 2006).

Considerable effort has been directed to the derivation of land cover information from remote sensing. At regional to global scales, for example, studies have developed from pioneering continental scale mapping programmes (e.g. Tucker et al. , 1985) to the situation today in which a variety of global maps are available (Herold et al., 2006; 2008). But many problems remain to be addressed. The large differences between maps of, apparently at least, the same phenomenon (Herold et al., 2006; See and Fritz, 2006; Potere et al., 2009) present user's with uncertainty over which, if any, to adopt (Herold et al., 2008; Shao and Wu, 2008). Unfortunately this situation is not aided by the poor attention sometimes paid to accuracy assessment, with many maps either not evaluated rigorously or only to a limited extent (Herold et al., 2006; Brannstrom et al., 2008). As a consequence of such problems there is a demand for information on map accuracy. 
The evaluation of map accuracy is now regarded as an important issue with accuracy assessment viewed by many as a fundamental component of mapping projects (Cihlar, 2000; Strahler et al., 2006). For those maps that have been evaluated, however, other problems often occur. Commonly, one problem is that remotely sensed land cover products are often viewed as failing to meet desired levels of accuracy (Townshend, 1992; Wilkinson, 1996; Gallego, 2004; Lu et al., 2008). This problem is particularly apparent in change detection based on post-classification comparison (Verbyla and Boles, 2000; Pontius and Lippitt, 2006). In such comparative assessments the amount of error in the classifications compared may obscure substantial change or could act to exaggerate change.

Thus, while remote sensing has considerable potential to provide information on land cover and its dynamics there is much work to be done before its full potential will be realised. Presently, there are many uncertainties with land cover change statistics (DeFries et al., 2002). For example, there are large differences, perhaps typically in the order of 5-10\%, between deforestation estimates derived from remote sensing and field measurement (Kintisch, 2007). Indeed, reappraisals of remote sensing estimates of land cover change have often taken place. For example, Skole and Tucker (1993) highlighted substantial, $50 \%$, over-estimation of deforestation arising through the use of coarse spatial resolution data sets and Achard et al. (2002) suggest that some estimates of deforestation rates in the humid tropics may have been exaggerated by $\sim 23 \%$. Similarly, other land cover changes may have been poorly and uncertainly evaluated. For example, recent estimates of the loss of Cerrado, a savanna that covered more than a fifth of Brazil 
and is a hotspot of biodiversity, vary greatly from 40 to 80\% (Jepson, 2005;

Brannstrom and Filippi, 2008). Problems linked to the accuracy of the mapping by remote sensing have been highlighted as a major concern and source of uncertainty in many studies.

This paper aims to explore some of the key issues associated with one major, yet rarely studied, error in the remote sensing of land cover change and the possibilities to combat this error source in order to enhance the utility of remote sensing as a reliable source of land cover data. Building on Foody (2009), attention is directed to the impacts of imperfect ground reference data on the accuracy of land cover change estimation, perceived and real. A key focus is on the magnitude and direction of biases introduced into derived estimates of change detection accuracy and change extent arising through the use of imperfect ground reference data. The paper also aims to illustrate and explore some of the possibilities to reduce the negative impacts that arise through the use of imperfect ground reference data. The imperfections considered include the presence of ground reference data error, known and unknown, as well as situations when ground reference data are absent. It will be shown that in some circumstances the negative impacts of ground reference data error can be accommodated so that corrected or refined estimates may be made. Additionally it will be demonstrated that accuracy may still sometimes be assessed and estimates of the extent of change made when ground reference data are absent; although some important concerns about a method used and its underlying assumptions are raised at the end of section 6. Throughout the focus is on 
some of the main scenarios encountered in remote sensing: the accuracy of change detection and the accuracy with which the amount or extent of change is estimated.

\section{Estimation from a binary confusion matrix}

Estimates of land cover change and of change detection accuracy are commonly made from a binary confusion matrix that illustrates the allocations to the change and nochange classes (Khorram, 1999; Congalton and Green, 2009). This approach has been widely used in a range of remote sensing studies (e.g. Woodcock et al., 2001; Chen et al., 2003; Stehman, 2005; Lunetta et al., 2006) and is the focus of this article. Critically, the distribution of entries in the confusion matrix together with the associated marginal values (row and column totals) may be used to derive numerous summary measures of the accuracy of the class allocations made and the amount of change that has occurred (Simon and Boring, 1990; Fielding and Bell, 1997; Khorram, 1999).

With interest on land cover change $(\Delta)$, the focus is on cases (e.g. pixels) for which there has been either a change $(\Delta=1)$ or no change $(\Delta=0)$ in the land cover class represented over the period studied. The probability that the ground information indicates change at a randomly selected location is $P(\Delta=1)=\theta$ and may be referred to as the prevalence of change. The output of the remote sensing change detection method is a binary classification in which a change is observed $(R=1)$ or not $(R=0)$. The accuracy with which change is detected and its extent estimated is derived from the confusion matrix 
that provides a cross-tabulation of the remote sensing derived labels with those contained in a corresponding ground reference data set.

Since there is no standardised way of presenting the confusion matrix it will be assumed throughout this paper that the columns of the matrix represent the ground reference data and the rows the classification derived by remote sensing (Figure 1). The matrix provides a summary of the class labelling for the $n$ cases used in a study, with each case lying within one of the matrix's four elements. The latter elements are represented by the entries $a$ to $d$ in Figure 1. For simplicity, it will also be assumed that a representative sample of cases was acquired by simple random sampling, although many other designs may be used (e.g. Stehman, 2009).

Cases lying in elements $a$ and $d$ of the confusion matrix are those for which the labelling in the two data sets agree; in some literature these are referred to as true positives and true negatives (Staquet et al., 1981; Fielding and Bell, 1997). For the cases in elements $b$ and $c$, however, the labelling in the two data sets differs; these are often referred to as false positives and false negatives (Staquet et al., 1981; Fielding and Bell, 1997). The relative frequency of cases in the matrix elements may be used to describe the degree of agreement between the two data sets. Indeed, the frequencies in elements $a$ to $d$ together with the marginal values of the matrix, $e$ to $h$, may be used to derive a set of quantitative estimates about the variable of study and degree of classification agreement or accuracy (Staquet et al., 1981; Fielding and Bell, 1997; Liu et al., 2009). Thus, for example, in the 
context of studies of land cover change, the confusion matrix provides information on the amount of land cover change and of change detection accuracy.

The accuracy of a binary classification is often described in terms of sensitivity and specificity (Rogan and Gladen, 1978; Staquet et al., 1981; Simon and Boring, 1990; Fielding and Bell, 1997). In the context of this article, the sensitivity of a classifier is the probability that the remote sensing method predicts change for a case of change, which can be expressed as the conditional probability $P(R=1 \mid \Delta=1)=S_{1}$ (Qu et al., 1996; Fielding and Bell, 1997). Thus, sensitivity is the proportion of cases correctly classified as having changed and may be derived from the confusion matrix from

$$
S_{1}=\frac{a}{a+c}=\frac{a}{e}
$$

The specificity of the classification is the probability of the remote sensing classifier predicting no-change for a case that has not changed, which can be expressed as the conditional probability $P(R=0 \mid \Delta=0)=S_{2}$. Specificity is, therefore, the proportion of cases correctly predicted to have not changed and may be derived from the confusion matrix using

$$
S_{2}=\frac{d}{b+d}=\frac{d}{f} \text {. }
$$


The sensitivity and specificity of the classification are, in the terminology used widely in the remote sensing literature, the producer's accuracy (Liu et al., 2009) for the change and no-change classes respectively.

Reading the matrix horizontally allows the derivation of two additional measures, often referred to as the predicted positive value and the predicted negative value (Simon and Boring, 1990; Fielding and Bell, 1997). The former may be calculated from the confusion matrix by

$$
U_{1}=\frac{a}{a+b}=\frac{a}{g}
$$

The predicted negative value may be derived from the confusion matrix using

$$
U_{2}=\frac{d}{c+d}=\frac{d}{h}
$$

The positive and negative predicted values for the classification are, in the terminology used widely in the remote sensing literature, the user's accuracy (Liu et al., 2009) for the change and no-change classes respectively.

Finally, one further measure that is often derived from the matrix is the prevalence, which may be derived from 


$$
\theta=\frac{a+c}{a+b+c+d}=\frac{e}{n}
$$

Other measures may be derived but are not considered here. Often the values derived from equations 1-5 are multiplied by 100 to yield a value as a percentage.

Attention is focused in this paper on the sensitivity (producer's accuracy for change) and prevalence (amount of change) estimates derived from the confusion matrix, although some comment will be made in relation to important issues connected with specificity and user's accuracy. This focus is mainly because the producer's accuracy and prevalence are typically of most interest in remote sensing studies but also because of relationships between the various measures. One key feature is that sensitivity (and specificity) is often viewed as being independent of prevalence (Rogan and Gladen, 1978; Staquet et al., 1981; Valenstein, 1990) but the positive and negative predicted values are a function of the quality of the classifier (indicted by its sensitivity and specificity) and the prevalence of change (Rogan and Gladen, 1978; Hui and Zhou, 1998; Simon and Boring, 1990; Enøe et al., 2001). The latter is evident in the expressions for $U_{1}$ and $U_{2}$ given in equations 6 and 7 respectively.

$$
\begin{aligned}
& U_{1}=\frac{S_{1} \theta}{S_{1} \theta+\left(1-S_{2}\right)(1-\theta)} \\
& U_{2}=\frac{S_{2}(1-\theta)}{S_{2}(1-\theta)+\left(1-S_{1}\right) \theta}
\end{aligned}
$$


As a consequence of these relationships the positive predictive value (user's accuracy of change) may be expected to increase if the prevalence of change increases (Staquet et al., 1981). In some disciplines, the prevalence dependency of $U_{1}$ and $U_{2}$ limits their value as general indices of classifier ability and accuracy as their magnitude has the undesirable property of fluctuating as a function of the variation in the variable under study. User's accuracy may still, however, be a useful measure of accuracy in studies of the remote sensing of land cover change, indicating one aspect of classification quality for the specific area under study.

Thusfar in this section it has been assumed implicitly that the ground reference data are error-free, representing a gold standard reference against which the results of the remote sensing classification may be evaluated. This assumption is unlikely to be satisfied. Moreover, it is known that even small errors in the reference data set can give rise to large bias in the accuracy measures and prevalence estimates derived from the confusion matrix (Vacek, 1985; Valenstein, 1990; Alonzo et al., 2002). Additionally, the specific manner in which the ground reference data errors are distributed among the matrix's elements can have a great impact of the nature of their effect. Ground reference data error can, for example, cause accuracy measures to be over- or under-estimated and so it is difficult to make simple generalizations of their effect (Hawkins et al., 2001). Moreover, one traditional approach to reducing this type of problem, through use of discrepant analysis, is problematic and may over-estimate classification quality (Miller, 1998; Hawkins et al., 2001). The impacts of ground reference data error, however, need to be 
recognised as they may lead to substantial misinterpretation of change detection accuracy and extent. This paper focuses on some of the negative impacts arising from the use of imperfect ground reference data and the methods that may be used to reduce them. It builds on recent work on imperfect ground reference data (e.g. van Oort, 2005; Bruzzone and Persello, 2009; Carlotto, 2009; Foody, 2009; Pontius and Li, 2009; Pontius and Petrova, 2010), addressing some of issues highlighted as requiring attention such as the impacts of varying types of imperfection, including correlated errors, and even missing ground reference data.

Since the ground reference data set is a binary classification that, like that derived by remote sensing, may contain error its quality can also be characterised by a sensitivity and specificity. To distinguish between the ground reference and remotely sensed classifications, the sensitivity and specificity of the ground reference data will be represented by $S_{1}^{\prime}$ and $S_{2}^{\prime}$ respectively. Furthermore, since the imperfections of the ground reference data set impact on the perceived accuracy of the remote sensing classification, the ${ }^{\wedge}$ symbol will placed over estimates derived from a confusion matrix

constructed with an imperfect ground reference data set (i.e., $\hat{S}_{1}$ is the estimate of the real sensitivity, $S_{1}$, that is derived when imperfect rather than perfect reference data are used).

\section{Ground data and their accuracy}

One key attribute of an ideal ground reference data set is that the class labels it contains are correct (100\% accuracy). Many ground reference data sets used in remote sensing 
may be deficient in relation to this attribute. Despite this situation, the ground reference data sets commonly used to evaluate the accuracy of land cover products derived from remote sensing are typically assumed to be correct or error-free (Foody, 2002; Carlotto, 2009).

The ground reference data used in a remote sensing project are unlikely to represent a gold standard as error may be contributed from a variety of sources. The latter include, for example, problems arising from mis-location of testing sites, presence of transitional classes, boundaries, typographical errors, restricted access to sites, uncertainties in class definition and temporal mismatches between image and field data acquisition (Powell et al., 2004; Comber et al., 2005; van Oort, 2005; See and Fritz, 2006; Thompson et al., 2007; Bradley, 2009). The problems may be especially apparent in studies of change as there is a need for ground reference data relating to at least two time periods for a phenomenon that is typically relatively rare (Stehman, 2009). As a result, obtaining ground data may be difficult, limiting both the quality of the data in terms of labelling accuracy as well as the number of cases and their location for use in accuracy assessment. Problems in obtaining ground data have often been reported in the literature (e.g. Liu and Zhou, 2004; Lu et al., 2008) and some even seek to work without ground data (Baraldi et al., 2005; Bruzzone and Marconcini, 2009). Moreover, it is sometimes noted that problems with the ground reference data may have negative impacts on the apparent accuracy of maps derived by remote sensing (Brannstrom and Filippi, 2008). Critically, ground reference data are often not error-free. Indeed the ground reference data set used is commonly just another classification in which cases may be mis-labelled but which is 
believed to be of higher quality than the remote sensing based classification that being evaluated (Stehman, 2009).

Although the accuracy of ground reference data is rarely known (Carlotto, 2009) a guide to the magnitude of error that may be present can be gleaned from the literature. For example, manual aerial photograph interpretation is often used as a source of ground reference data. Studies of inter-interpreter agreement in the analysis of imagery highlight, however, substantial disagreement in labelling, even when using fine spatial resolution imagery (e.g. very large scale aerial photography) and trained interpreters as a source of ground reference data. While the magnitude of the errors may be expected to vary from project to project (e.g. as a function of image properties, thematic resolution interpreter training and experience etc.) the magnitude of disagreement may be large. Powell et al. (2004), for example, report that interpreters disagreed on $30 \%$ of cases. Other studies have also shown considerable differences in class allocations. For example, in relation to data acquired by aerial photograph interpretation, Thompson et al. (2007) found differences in labels for detailed forest classes for $64 \%$ of sites evaluated and Johnson and Ross (2008) report up to $40 \%$ disagreement in labelling. Error is, therefore, often recognised to be substantial in ground reference data derived from aerial photograph interpretation (Mannel et al., 2006; Xu et al., 2009). An additional guide to the quality of ground data is evident from the common use of a classification of fine spatial resolution satellite sensor data to act as ground data for use in the validation of a coarser spatial resolution product (Justice et al., 2000; Foody, 2002; Brannstrom and Filippi, 2008; Brannstrom et al., 2008). Here, a key feature is that a common objective is to map the 
land cover to a target accuracy of $\sim 85 \%$ (Weng, 2002; Yang and Lo, 2002; Rogan et al., 2003; Treitz and Rogan, 2004; Yang and Liu, 2005; Mundia and Aniya, 2005). Not only do many studies fail to achieve such accuracy (Wilkinson, 2005; Shao and Wu, 2008) but they are also implicitly stating that a $\sim 15 \%$ error in the ground data set is tolerable. It should be recognised that the use of such sub-optimal reference data is also a reflection of the costs of acquiring high quality data. For example, it may be prohibitively costly to undertake a large programme of fieldwork or to acquire very fine spatial resolution imagery and so researchers are effectively compelled to use less than ideal data for reference purposes.

It seems, therefore, that researchers sometimes use ground reference data sets that may contain substantial error even though it is well known that error in the ground reference data can be a problem and limit the value of the confusion matrix and measures derived from it (Congalton, 1991; Foody, 2002; Powell et al., 2004; Mann and Rothley, 2006). This situation may have arisen because researchers are unaware of the size and nature of the problems arising from the use of an imperfect ground reference data set and/or of how to use such information constructively. However, it must be recognised that errors in the ground reference data set may greatly limit studies. Bearing in mind that change is typically rare, the error in the two data sets used to construct the confusion matrix may mean that they are of insufficient accuracy to detect let alone accurately quantify change (Pontius and Lippitt, 2006). Consequently, it is important that ground reference data be scrutinised carefully and the implications of imperfections considered (Powell et al., 2006; Kennedy et al., 2009) as information on their quality can enhance studies. 
Critically, ground reference data error should not be ignored, no matter how convenient it may sometimes be to do so, as its effects may be substantial and may possibly be correctable (Carlotto, 2009; Foody, 2009). There is, therefore, a need to understand the possible effects of ground reference data error and gain an appreciation of how to correct for them.

\section{Data}

Simulated data were mainly used in order to remove complexities and uncertainties linked to the impacts of sources of error other than ground reference data error. A series of data sets were generated to explore the impacts of imperfect ground reference data on the perceived accuracy of change detection and change extent as well as illustrate possible methods to correct for the effects of ground reference data error. Here, three different groups of data sets were formed to allow evaluation of the effect of and correction for a variety of ground data imperfections on studies of land cover change. The data sets were designed to allow a spectrum of imperfections to be addressed. The imperfections studied ranged from the situation in which the errors in the ground and remotely sensed data were independent and the amount of ground reference data error known, through the situation in which the error was known but the errors correlated between the two data sets cross-tabulated, to the situation in which there was not only no information on ground reference data error but no ground reference data at all. With the latter there was no ground reference data error or corrective strategy to consider but 
rather the problem of deriving information on the accuracy of land cover change estimates without the ability to consult ground data.

The simplest situation arises when the errors used in the data sets to form the confusion matrix are uncorrelated. This situation is often assumed. For example, errors have often been assumed to be independent in discussion of error propagation in post-classification change detection (Pontius and Lippitt, 2006). Additionally, ensemble approaches used to increase classification accuracy also commonly assume that the classifiers used have independent errors (Bruzzone et al., 2004). To explore issues connected with the use of an imperfect ground reference data set when the errors are independent from those in the remotely sensed data set, a series of binary confusion matrices were formed to represent scenarios arising from the cross-tabulation of classification outputs of known quality. The formation of a confusion matrix required the specification of the sensitivity and specificity of each classification and the prevalence. It was assumed here that change was relatively rare to reflect real remote sensing situations. Two levels of prevalence were considered: $20 \%$ and $5 \%$. These levels were defined relatively arbitrarily but lie within the range of change often reported in the literature (Pontius and Lippitt, 2006). In specifying the accuracy of the two data sets used to form a confusion matrix it was also assumed that the ground reference data set was at least as accurate, but normally more accurate, than remote sensing method. The values for sensitivity and specificity used were also defined relatively arbitrarily but selected to fit with typical remote sensing situations in which a common target in mapping, albeit of debatable suitability (Foody, 2008 ), is $\sim 85 \%$ and knowledge that the accuracies reported for land cover mapping in the 
literature are typically $>65 \%$ (Wilkinson, 2005). In keeping with a common desire to classify classes to similar accuracy, it was assumed that the remote sensing technique had equal sensitivity and specificity although an example with unequal settings is also illustrated. Finally, a sample size of 1000 was used throughout and assumed to have been drawn by simple random sampling. The key details of the scenarios used are illustrated in Table 1. Since the properties of each scenario were known it was possible to construct the confusion matrix that would have been derived from the use of perfect ground reference data $\left(S_{1}^{\prime}=S_{2}^{\prime}=100 \%\right)$ as well as that arising from the use of the imperfect ground reference data (Figure 2).

Sometimes the errors in the ground and image classification are correlated. Correlated errors may arise in a number of ways and are sometimes noted in the remote sensing literature (Congalton, 1988; van Oort, 2005, 2007). For example, correlated errors may be expected if the classifiers have a similar basis and so tend to err on the same cases. Since correlated errors have a different impact to errors that are independent it is important to know the nature of the errors in the data sets used. Two classifications may be considered to be conditionally independent when the sensitivity (specificity) of one classification does not depend on the outcome of the other (Gardner et al., 2000; Georgiadis et al., 2003). The degree of dependence may be assessed using a measure such as the conditional correlation between the classification outcomes. For example, if the conditional correlation between classification outcomes differs substantially from zero, the classifications may be considered conditionally dependent. The conditional correlation between classification outcomes may be determined from 


$$
\rho_{1}=\frac{S_{1}^{*}-S_{1}^{1} S_{1}^{2}}{\sqrt{S_{1}^{1}\left(1-S_{1}^{1}\right) S_{1}^{2}\left(1-S_{1}^{2}\right)}}
$$

for change and

$$
\rho_{0}=\frac{S_{2}^{*}-S_{2}^{1} S_{2}^{2}}{\sqrt{S_{2}^{1}\left(1-S_{2}^{1}\right) S_{2}^{2}\left(1-S_{2}^{2}\right)}}
$$

for no-change. where the super-scripts 1 and 2 indicate the two classifications undercomparison, $S_{1}^{*}=P\left(R^{1}=1, R^{2}=1 \mid \Delta=1\right)$ and $S_{2}^{*}=P\left(R^{1}=0, R^{2}=0 \mid \Delta=0\right)$. Further details on conditional dependence are given in the literature (e.g. Vacek, 1985; Qu et al., 1996; Branscum et al., 2005; Georgiadis et al., 2005). This paper focuses on situations in which the errors between the cross-classified data forming a confusion matrix are independent (i.e. conditional independence exists) and when they are correlated (i.e. conditional dependence exists).

To illustrate the impacts arising from the situation in which the ground reference data errors were correlated with those in the remotely sensed data set, a further series of data sets were generated. Using the confusion matrix defined with perfect ground data associated with scenario B as base (see right hand column for scenario B in Figure 2), three further scenarios were simulated by adjusting the matrix elements to reflect varying amounts of correlated error. For example, if the errors in the remote sensing classification and ground reference data occur with the same cases and the ground reference data set 
had $S_{1}^{\prime}=S_{2}^{\prime}=\gamma \%$, then $100-\gamma \%$ of cases of change should be misclassified as no-change in both classifications and $100-\gamma \%$ of no-change cases should be misclassified as belonging to the change class in both classifications; see Valenstein (1990) for an example. Here, three scenarios, F, G and H, were simulated with $100-\gamma \%$ set at 1.0, 2.0 and $10.0 \%$ respectively (Figure 3 ).

As a further guide to the impacts of imperfect reference data on the accuracy of the remote sensing of land cover change, the effect of reference data errors on two real confusion matrices was evaluated. This evaluation should help to ensure that the study has direct relevance to real situations, particularly with regard to actual levels of producer's and user's accuracy as well as prevalence encountered in studies. Here, the focus was on two matrices discussed in Stehman (2005; relating to what were termed map A and map B in Table 2, page 471 and based on papers by Chen et al. (2003) and Woodcock et al. (2001) respectively); these data do not relate in any way to those for scenarios A and B discussed previously. To aid comparison with the other analyses, the selected matrices were adjusted to allow presentation in the same style as others in this paper on the assumption of a sample size of 1000 (Figure 4). Both of the matrices show highly accurate classifications, with overall accuracy values of $90.8 \%$ and $95.8 \%$ for maps A and B respectively. The two matrices, however, differ greatly in detail, notably with regard to the producer's and user's accuracy of change. For the purposes of this paper it was assumed that each of the two matrices had been derived with the use of a gold standard ground reference data set. The impacts of ground reference data on the remote sensing of change were then simulated by adding errors into the representations. 
In keeping with the desire to use a reference data set that is more accurate than the data set it is being used to evaluate, the errors introduced were small. Specifically, with map A a new matrix was simulated for the situation in which the ground reference data set was 95\% accurate while for map B the accuracy was 98\%; in each case it was assumed, for simplicity, that the sensitivity and specificity of the reference data classification were equal. The simulation was undertaken in the same fashion as described above for independent and for correlated errors yielding new matrices based on imperfect reference data (Figure 4).

Further data sets were generated to illustrate land cover change estimation when ground reference data were absent, a particularly extreme case of imperfect ground reference data. In this case it was insufficient to generate just a confusion matrix as information on the class allocation for each case in each classification was required. Additionally, the methods used required information from more than one classifier. The data relating to scenario B (Table 1, Figure 2) were used as a base. A data set of 1000 cases that when cross-tabulated would match the results based on analyses of scenario B was formed. Data for three additional scenarios, I, J and $\mathrm{K}$, were generated in such a way that allocations to the change and no-change class were made randomly in accordance to a specified probability of allocation to the correct class (defined with regard to the perfect ground reference data). The probabilities used were $0.90,0.85$ and 0.70 for scenarios I, J and $\mathrm{K}$ respectively; the nature of the actual class allocations derived from this process and accuracy of each of these three scenarios is evident in the confusion matrices derived (Figure 5). The three new classifications were derived, therefore, in a way that should 
ensure that the errors contained were independent of each other and that represented by the output of the remote sensing classifier defined by scenario B. Indeed, the only major difference between the four classifications was the magnitude of accuracy.

Finally, a further data set was generated to illustrate a method that may be used when the errors are both unknown and correlated. For this, the remotely sensed classification in scenario $\mathrm{J}$ was used as a base. A new data set was generated by copying the data in scenario $\mathrm{J}$ and then manually changing the class labels for 150 cases (50 cases that had actually changed and 100 cases that had not). In this way the new data set, forming scenario L, which was highly correlated to the data in scenario $\mathrm{J}$ but of a slightly lower accuracy was formed (Figure 5). The overall level of agreement between the classifications in scenarios $\mathrm{J}$ and $\mathrm{L}$ was high, with agreement in labelling noted for $85 \%$ of the cases. Moreover, the magnitude of the conditional correlations between the data in scenarios $\mathbf{J}$ and $\mathrm{L}$ derived from equations 8 and 9 was large, substantially larger than zero and the value observed for other comparisons (Table 2).

\section{Methods}

The data sets generated were used to explore the impacts of imperfect ground reference data on the perceived accuracy of change detection and change extent estimation as well as illustrate the potential of methods to reduce them. Initial focus was directed on an exploration of the magnitude and direction of errors introduced by the use of an imperfect ground reference data set which was illustrated by comparison of confusion matrices and 
derived estimates. Additionally, as the properties of the two classifications crosstabulated to form each confusion matrix were known, it was possible to model the variation in the perceived accuracy of change detection as a function of the accuracy of the prevalence of change. That is, the known values of the sensitivity and specificity for each classification at a specified value of the true prevalence allow the derivation of the apparent sensitivity and specificity. Assuming the data sets to be conditionally independent, this was achieved using equations 10 and 11 (Gart and Buck, 1966) for the perceived values of sensitivity

$$
\hat{S}_{1}=\frac{\left(1-S_{2}^{\prime}\right)\left(1-S_{2}\right)+\theta\left(S_{1}^{\prime} S_{1}-\left(1-S_{2}^{\prime}\right)\left(1-S_{2}\right)\right)}{\left(1-S_{2}^{\prime}\right)+\theta\left(S_{1}^{\prime}+S_{2}^{\prime}-1\right)}
$$

and for specificity

$$
\hat{S}_{2}=\frac{S_{2}^{\prime} S_{2}+\theta\left(\left(1-S_{1}^{\prime}\right)\left(1-S_{1}\right)-S_{2}^{\prime} S_{2}\right)}{S_{2}^{\prime}-\theta\left(S_{1}^{\prime}+S_{2}^{\prime}-1\right)} \quad .
$$

After illustrating the impacts of ground reference data error on the accuracy of change detection and extent estimation, attention turned toward an evaluation of the methods to reduce or even remove them. A variety of approaches exist to adjust estimates for known or even unknown (Hui and Zhou, 1998; Enøe et al., 2000) reference data errors. These approaches differ greatly but two broad categories that have relevance to common remote sensing scenarios are considered here. The first relates to the situation in which a single classification illustrating change and no-change has been undertaken and is validated 
against an imperfect ground reference data set of known accuracy. In recognition of the problems of obtaining information on ground reference data accuracy and to illustrate additional features, the second relates to the situation when there is no ground reference data but class allocation information from multiple classifications is available. This latter approach is based on latent class analysis.

\subsection{Single classification and known ground data error}

Although the quality of ground reference data will often be unknown it may sometimes be possible to estimate their accuracy (e.g. on the basis of prior experience or from acquisition of additional field based information $e t c$.). If the accuracy of the ground reference data set is known and its errors are conditionally independent from those in the remote sensing based classification it is possible to derive the real change detection accuracy and extent of change from the observed confusion matrix. The correction for ground reference data error in this situation is derived algebraically with the real change detection accuracy and change extent derivable from simple equations (Gart and Buck, 1966; Rogan and Gladen, 1978; Staquet et al., 1981; Miller, 1998; Enøe et al., 2000). For the confusion matrix defined in Figure 1, the real producer's accuracy of change may be derived from

$$
S_{1}=\frac{g S_{2}^{\prime}-b}{n\left(S_{2}^{\prime}-1\right)+e} .
$$

Similarly, the real user's accuracy may be derived from, 


$$
U_{1}=\frac{S_{1}\left(e-n+S_{2}^{\prime} n\right)}{g\left(S_{1}^{\prime}+S_{2}^{\prime}-1\right)}
$$

Finally, a the real prevalence or amount of the extent of change may be derived from

$$
\theta=\frac{n\left(S_{2}^{\prime}-1\right)+e}{n\left(S_{1}^{\prime}+S_{2}^{\prime}-1\right)}
$$

Further details, including a discussion on the derivation of the relationships and formulae for standard errors of the estimates are given in the literature (e.g. Gart and Buck, 1966; Messam et al., 2008). The key concern for this article, however, is that, while ground reference data error is undesirable and can have substantial negative impacts on land cover change estimates researchers can do something about it.

Equations 12-14, and similar, should not be used if the conditional independence assumption is violated as this can produce extremely biased results. In particular, if the conditional independence assumption is violated, the classification error rates may be underestimated (Vacek, 1985; Enøe et al., 2000). Estimates of prevalence may also be biased but in a direction that is dependent on the exact circumstances. However, in many cases it may be expected that a low prevalence would be over-estimated while a high prevalence under-estimated (Vacek, 1985). Thus, when the conditional independence assumption cannot be made researchers may need to adopt an alternative approach to address the impacts of imperfect ground reference data and one possibility is discussed below. 


\subsection{Latent class analysis}

The methods to correct estimates for ground reference data error defined by equations 1214 may be used when the conditional independence assumption holds and the quality of the reference classification, expressed in terms of sensitivity and specificity, are known. However, both conditions may be hard to satisfy. Fortunately a range of alternative approaches exist to accommodate for the effects of ground reference data error (Espeland and Handelman, 1989; Qu et al., 1996; Hui and Zhou, 1998; Enøe et al., 2000). These approaches vary from methods that may be suited to situations when some properties of the ground reference data quality are known through to situations when there is no information on the quality of the ground reference data set and conditional independence cannot be assumed (Staquet et al., 1981; Hui and Zhou, 1998; Enøe et al., 2000). Indeed methods exist for extreme cases of imperfect ground reference data, such as when ground reference data are absent (Qu and Hadgu, 1998).

With no information on the quality of the ground reference data available more information or data on the problem than that contained in a single confusion matrix is required in order to allow reliable inferences to be drawn (Hui and Zhou, 1998; Enøe et al., 2000). This can be achieved in a variety of ways, notably by applying multiple classifiers to the data or by application of the classification(s) to more than one population (Hui and Zhou, 1998; Georgiadis et al., 2003). Of these, the application of multiple classifiers is perhaps the most suited to studies of the remote sensing of land cover change. Indeed the application of multiple classifications is popular as a means of 
increasing the accuracy of classification through use of ensemble methods (Bruzzone $e t$ al., 2004).

The accuracy of a classification and estimates of prevalence can often be made in the absence of a perfect or gold standard reference data set through the use of a latent class model (Espeland and Handelman, 1989; Qu et al., 1996; Goethebeur et al., 2000; Enøe et al., 2001). Aside from one previous study on accuracy assessment (Patil and Taille, 2003), latent class models do not appear to have been used in remote sensing research and so some general background will be provided before presenting the models used.

In a standard latent class analysis it is assumed that the remote sensing based classifications undertaken are imperfect indicators of the unobserved (latent) status of change but that the observed associations among the classification outputs, that may be of unknown sensitivity and specificity, can be explained by the latent variable (Rindskopf and Rindskopf, 1986; Yang and Becker, 1997; Engels et al., 2000). Moreover, the assumption of conditional independence that underlies the standard latent class model means that its only parameters are the latent class probabilities and the conditional probabilities, which define the sensitivity and specificity of the classifications (Yang and Becker, 1997; Rindskopf, 2002). Related methods may also be used when the assumption of conditional independence cannot be made. 
A standard latent class model involving a single latent variable with two latent classes (change and no-change) and based upon the use of four independent classifiers, W, X, Y and Z, whose outputs are labels $w, x, y, z=0,1$ is based on

$$
\pi_{w x y z t}=\pi_{t}^{\Delta} \pi_{w x y z t}^{w X Y \mid \Delta}
$$

with

$$
\pi_{w x y z t}^{W X Y Z \mid \Delta}=\pi_{w t}^{W \mid \Delta} \pi_{x t}^{X \mid \Delta} \pi_{y t}^{Y \mid \Delta} \pi_{z t}^{Z \mid \Delta}
$$

where $\pi_{w x y z t}^{W X Y \mid \Delta}$ is the conditional probability that the pattern of class labels derived from the classifiers is $(w, x, y, z)$ given that the case has a change status $t(1$ or 0$)$ and $\pi_{t}^{\Delta}$ is the probability that a case has the change status $t$ (Vermunt, 1997; Yang and Becker, 1997); note the classification outputs are variables in the analysis and so written in italics in the equations. Moreover, the conditional probabilities that represent the sensitivity and specificity of each classifier are parameters of the model (e.g. $\pi_{11}^{W \mid \Delta}$ is the sensitivity of classifier W). The fit of a latent class model to the data is often evaluated with regard to a measure such as the likelihood ratio chi-squared statistic, $L^{2}$; with a model normally viewed as fitting the data if the value of $L^{2}$ is sufficiently small to be attributable to the effect of chance (Magidson and Vermunt, 2004).

The basic latent class model may also be represented as a log-linear model from which it is possible to estimate the sensitivity and specificity of classifications as well as the 
prevalence (Espeland and Handelman, 1989; Hui and Zhou, 1998). For example, equation 16 is equivalent to the latent class log-linear model represented by equation 17 ,

$$
\log \pi_{w x y z}^{W X Y \Delta}=\lambda_{t}^{\Delta}+\lambda_{w t}^{W \Delta}+\lambda_{x t}^{X \Delta}+\lambda_{y t}^{Y \Delta}+\lambda_{z t}^{Z \Delta}
$$

where $\lambda$ are the main effects of the true change status and the predictions made by the three classifiers (Hui and Zhou, 1998). As above, the sensitivity and specificity are directly related to model parameters and the prevalence of change is estimated as the proportion of the sample estimated to have changed in the latent variable (Espeland and Handelman, 1989).

The model components represented in equations 16 and 17 may also be adapted to allow for situations in which the assumption of conditional independence is untenable. For example, if classifiers $\mathrm{Y}$ and $\mathrm{Z}$ were not independent the model would use

$$
\pi_{w x y z t}^{W X Y Z \mid \Delta}=\pi_{w t}^{W \mid \Delta} \pi_{x t}^{X \mid \Delta} \pi_{y z t}^{Y Z \mid \Delta}
$$

or, as the log-linear model,

$$
\log \pi_{w x y z t}^{W X Y Z \Delta}=\lambda_{t}^{\Delta}+\lambda_{w t}^{W \Delta}+\lambda_{x t}^{X \Delta}+\lambda_{y t}^{Y \Delta}+\lambda_{z t}^{Z \Delta}+\lambda_{y z t}^{Y Z \Delta}
$$

in which the final parameter in equations 18 and 19 represents the dependence between $\mathrm{Y}$ and Z. Although the inclusion of the latter term allows application when the assumption 
of conditional independence is inappropriate its presence also means that the model's parameters (e.g. $\lambda_{y t}^{Y \Delta}$ and $\lambda_{z t}^{Z \Delta}$ in equation 19) no longer have a direct interpretation in terms of sensitivity and specificity for classifiers Y and Z (Yang and Becker, 1997; Hui and Zhou, 1998). Yang and Becker (1997) proposed parameterizing the log-linear model in marginal models in which a direct relation to sensitivity and specificity may still be made. Equation 19 is equivalent to a latent class marginal model that allows for dependence between classifiers Y and Z (Yang and Becker, 1997; Becker and Yang, 1998). In such a model the univariate marginal logits are directly related to sensitivity and specificity. Thus, for example, the sensitivity of classification Y may be estimated from

$$
S_{1}=\frac{1}{1+e^{\alpha_{1}^{Y}}}
$$

where $e^{\alpha_{1}^{Y}}=\frac{\pi_{01}^{Y \mid \Delta}}{\pi_{11}^{Y \mid \Delta}}$ and its specificity from

$$
S_{2}=\frac{e^{\alpha_{0}^{Y}}}{1+e^{\alpha_{0}^{Y}}}
$$

where $e^{\alpha_{0}^{Y}}=\frac{\pi_{00}^{Y \mid \Delta}}{\pi_{10}^{Y \mid \Delta}}$ (Yang and Becker, 1997; Hui and Zhou, 1998). Critically, it is evident that the latent class based method may be applied when the variables are conditionally independent or conditionally dependent. 
A test for conditional independence should be undertaken to ensure an appropriate model is used. A variety of approaches have been reported in the literature for assessing conditional independence. Here, a modified version of the log-odds ratio check method (Garrett and Zeger, 2000), implemented using the CONDEP programme (http://www.john-uebersax.com/stat/condep.html), was used. The log-odds ratio check method is based on a comparison of the log-odds ratio for the observed $\left(\psi_{\mathrm{o}}\right)$ and expected $\left(\psi_{\mathrm{e}}\right)$ data, and the evaluation was based upon the comparison in terms of the $\mathrm{z}$ score

$$
z=\frac{\psi_{o}-\psi_{e}}{\sigma_{\psi_{e}}}
$$

and hence a value of $z$ above the selected critical value indicates conditional dependence (e.g. for a two-sided test at the 0.05 level of significance the critical value is $|1.96|$ ).

Here, latent class models were used to illustrate the potential to assess the accuracy of change detection and derive estimates of the extent of change when ground reference data were absent. This assessment was undertaken for the situation in which the classifications were conditionally independent and when they were conditionally dependent. First, using just the outputs from the remote sensing change detection classifiers defined in scenarios $\mathrm{B}$, I, J and $\mathrm{K}$, which were created in a manner that should ensure independence of errors, the approach represented by equations 15 and 16 was undertaken. Specifically, the model employed

$$
\pi_{b i j k t}^{B I J \mid \Delta}=\pi_{b t}^{B \mid \Delta} \pi_{i t}^{I \mid \Delta} \pi_{j t}^{J \mid \Delta} \pi_{k t}^{K \mid \Delta}
$$


and then was used to allow the producer's accuracy (sensitivity) of each classifier and the prevalence of change to be estimated.

Finally, to illustrate the potential of the latent class modelling approach when correlated errors occur, a further analysis was undertaken. For this, the data in scenario $\mathrm{K}$ were replaced by those in scenario $\mathrm{L}$, which was highly correlated with the data in scenario $\mathrm{J}$. Then, the latent class model using

$$
\pi_{b i j l t}^{B I J \mid \Delta}=\pi_{b t}^{B \mid \Delta} \pi_{i t}^{I \mid \Delta} \pi_{j l t}^{J L \mid \Delta}
$$

was solved. The analyses based on equations 23 and 24 were undertaken with the LEM software (Vermunt 1997; software available at http://www.uvt.nl/faculteiten/fsw/organisatie/departementen/mto/software2.html). Some further analyses in which different dependence structures were specified were undertaken to explore the latent class modelling approach with these data.

A series of products may be derived from the latent class analysis, including information on class membership per-case that allows a confusion matrix to be generated from which a variety of accuracy measures could be derived. Here, however, attention is on the producer's accuracy and prevalence which are parameters of the basic model. Critically, it should be noted that the results from the use of both latent class models founded on equations 23 and 24 were derived without use of ground reference data. In each case, 
however, the actual accuracy of the classification and amount of change was known as the data had been simulated with known properties. Finally, however, it is important to note that the focus in this paper is on the potential of the latent class analysis using simulated data of known properties for which it is relatively simple to define appropriate models. Real world applications may present a range of challenges and the validity of the approach and the ability to use it effectively requires further investigation. One critical feature is that, as a model based approach, it is important that the underlying assumptions of the model are satisfied.

\section{Results and Discussion}

Error in the ground reference data set impacted greatly on the perceived accuracy of change detection and of the amount of change that appeared to occur. The nature of the effect of ground reference data error varied greatly, especially in relation to whether the errors in the cross-tabulated data sets were independent or correlated. It may, however, be possible to correct for the negative effects of ground reference data error. The latter will be discussed after first evaluating the impacts arising from the use of an imperfect reference data set.

\subsection{Independent errors.}

For scenarios A-E it was apparent that ground reference data error introduced considerable, and often systematic, bias into the estimates of change detection accuracy and extent (Figure 2). In particular, it was evident that the producer's accuracy of change 
was generally underestimated while the extent of change was over-estimated; of the scenarios investigated only scenario D did not follow this trend. Moreover, the magnitude of the bias introduced was large even when the ground reference data set was of very high accuracy. For example, in scenario A the ground reference data set was $95 \%$ accurate but caused a $13.9 \%$ underestimation of the producer's accuracy (the perceived producer's accuracy, $\hat{S}_{1}$, was estimated to be $76.1 \%$ but actually the real value, $S_{1}$, was $90.0 \%)$. Not only was the magnitude of the bias large but it also had the effect of reducing the perceived accuracy below the popular $85 \%$ target, potentially leading to a highly accurate classification being wrongly rejected as not meeting the required standard. Note also that the magnitude of the bias in producer's accuracy increased with the amount of error in the ground reference data set, as evident from the results associated with scenarios $\mathrm{A}, \mathrm{B}$ and $\mathrm{C}$.

The prevalence or amount of change was also mis-estimated through the use of an imperfect ground reference data set. In all scenarios, the prevalence was over-estimated, rising from an estimate that was $3.0 \%$ larger than reality for scenario A to an $18.0 \%$ overestimation for scenario C. Indeed, in the latter scenario the amount of change was estimated to be nearly twice that which actually occurred.

It was also evident that the prevalence of change had an impact on the results. This is especially apparent in relation to scenarios B and E which differed only in the prevalence of change. It was apparent that at low prevalence, scenario E, the producer's accuracy was grossly underestimated; the perceived producer's accuracy, $\hat{S}_{1}$, was estimated to be 
$39.3 \%$, roughly half of the actual value of $80.0 \%$. Additionally, the prevalence of change was substantially over-estimated, more so than with scenario B. Thus the accuracy of change detection by remote sensing and of change extent estimation was a function of the amount of change that has occurred; the estimates are prevalence-dependent. Moreover, the effect of variation in prevalence was modelled and revealed considerable impacts on the magnitude of the perceived accuracy (Gart and Buck, 1996). The relationship between the observed or perceived accuracy with prevalence is highlighted for the scenarios discussed in Figure 6. Note, for example, that the producer's accuracy could vary greatly with prevalence. The producer's accuracy was only independent of prevalence for scenario $\mathrm{D}$.

The results from scenario D generally differed from the others. Scenario D was unusual in that the classification used as ground reference data had a perfect specificity. The consequence of this was that, for scenario D, the producer's accuracy was estimated correctly. Moreover, the estimate of producer's accuracy was not dependent on prevalence (Figure 6). Note that if the ground reference data set had possessed a perfect sensitivity but an imperfect specificity then the producer's accuracy of change for scenario D would have varied with prevalence while the corresponding accuracy for the no-change class would have been prevalent independent. Scenario D is particularly useful in highlighting that the effects of ground data error vary greatly dependent on how the errors are distributed amongst the confusion matrix elements, which is a function of the sensitivity and specificity of the classifications cross-tabulated. 
Although not a major concern to this paper, it was also evident that substantial bias could be introduced by ground reference data error into the estimation of user's accuracy. With user's accuracy, the effect of ground reference data error varied in magnitude and the direction of the bias introduced also varied. For example, scenarios $\mathrm{A}$ and $\mathrm{C}$ show, respectively, under-estimation and over-estimation of user's accuracy while the estimate for scenario B was correct (Figure 2).

\subsection{Correlated errors}

Ground reference data error sometimes caused substantial bias to the accuracy and extent estimates derived from a confusion matrix when there was correlation in the errors in the

data sets cross-tabulated (Figure 3). The pattern in the results was, however, dissimilar to the general trend obtained with uncorrelated errors (Figure 2). Note, for example, that with correlated errors the producer's accuracy was systematically mis-estimated but in the opposite direction to that generally observed with uncorrelated errors. Additionally, it was evident that the magnitude of the over-estimation of the producer's accuracy was positively related to the degree of error, rising from scenario $\mathrm{F}$ through $\mathrm{G}$ to $\mathrm{H}$ (Figure 3).

The prevalence of change was also mis-estimated as a consequence of ground reference data error. For scenarios $\mathrm{F}$ to $\mathrm{H}$, the prevalence of change was consistently overestimated. The bias introduced by ground reference data error into estimates of the user's accuracy could be large, with $U_{1}$ increasing from $50.0 \%$ to $75.0 \%$ in the scenarios evaluated (Figure 3). 
Errors that are correlated between the two data sets cross-tabulated to form a confusion matrix, therefore, impact differently to uncorrelated errors. Note, for example, the differences between scenarios $\mathrm{B}$ and $\mathrm{H}$ which were dissimilar in terms of the correlation between the data sets (Figures 2 and 3). Critically, each shows the impact of a $10 \%$ error in the ground reference data set. With both, the prevalence was over-estimated but in scenario B the producer's accuracy for change was under-estimated by $18.5 \%$ while it was over-estimated by $12.3 \%$ in scenario H. Understanding the impacts of ground reference data error, therefore, requires information on the nature of the error and, in particular, the degree of correlation between the errors in the ground reference and image classification data sets used to form the confusion matrix.

\subsubsection{Evaluation based on real matrices}

Imperfect reference data had a large impact on the estimates derived from the analyses based on the two real confusion matrices (Figure 4). The trends observed were similar to those reported above. For example, in relation to both map A and map B the producer's and user's accuracy were under-estimated when independent errors occurred but overestimated in the presence of correlated errors. Note also that the magnitude of misestimation could sometimes be substantial even though all of the data sets used were of very high accuracy. For example, in relation to map A, the use of reference data with 5\% error could yield an under-estimation of the producer's accuracy of $13.5 \%$ if the errors were independent and an over-estimation of the user's accuracy by $22.4 \%$ if the errors were correlated. It was, therefore, evident that substantial mis-estimation of accuracy and change extent may be made as a consequence of using reference data containing on small 
amounts of error (Figure 4). Note also that the impacts affect other features. For example, the overall accuracy of a classification may be substantially mis-estimated (e.g. map A has an accuracy of $90.8 \%$ but appears to be $86.8 \%$ if errors are independent and $95.8 \%$ if errors are correlated). Moreover, the results from the analyses of the simulated data discussed above suggest that larger impacts would have been observed if the prevalence of change had been smaller than the relatively high values that had been recorded. The results highlight the need to address the effects of imperfect ground reference data in studies of land cover change by remote sensing.

\subsubsection{Discussion}

A key result highlighted above was that the use of imperfect ground reference data may sometimes result in a substantial mis-estimation of the amount of change, and so be a source of error contributing to the inaccuracy of change statistics reported in the literature. Ground data error also impacted greatly on the apparent accuracy of change detection. The impacts of ground reference data error varied as a function of the nature of the errors, notably in terms of their absolute and relative magnitude as well as direction of the bias introduced into estimates. Although there were some circumstances in which ground reference data error had no or only a small effect (e.g. on the estimation of producer's accuracy in scenario D) they typically result in a false impression of classification accuracy and extent of change. Critically, however, the use of imperfect ground reference data can be a source of substantial error in studies of land cover change. Even when the ground reference data were very accurate the magnitude of the misestimation of accuracy and extent could be large so the problems of ground data quality 
should not be ignored. Indeed the problems of ground data quality should be considered in the design of a study, such as when planning the sample size of ground reference data sets (Rahme and Joseph, 1998; Messam et al., 2008) and in the interpretation of results. For example, the desire to sometimes focus attention disproportionately to hotspots of change (e.g. Broich et al., 2009) may result in problems linked to the prevalence dependency of some accuracy measures. The results above highlighted, for instance, that the use of an imperfect ground reference data set may result in the apparent accuracy of change detection varying from location to location as a function of the prevalence of change. A classifier that was highly accurate at a location with considerable change may appear to be of low accuracy when applied to a location with little change. This problem could be interpreted as a failing of the classifier or perhaps the result of as some transferability problem but may, at least in part, be a function of the use of imperfect ground reference data. The various problems of ground reference data quality noted reinforce the oft-stated call for the term ground truth to be avoided. Although truth is a concept and open to interpretation, the term ground truth may imply to some that the ground data set is error-free when this is unlikely and the imperfections of the ground reference data set, even if minor, can be a source of considerable error and misinterpretation.

\subsection{Correcting for ground reference data error}

For the situation in which the errors are independent and the quality of the ground reference data set is known, equations 12-14 may be used to derive the actual properties from the observed or perceived values. Thus, the application of equations 12-14 to the 
observed data in Figure 2 yields the real values, which are known as the data were simulated with known properties. That is, the estimate derived from the confusion matrix formed with regard to the imperfect ground reference data (e.g. $\hat{S}_{1}$ ) may be used to derive the real value (e.g. $S_{1}$ ) through the use of the appropriate equation. These results reinforce calls made in other studies to use the confusion matrix for more than just a description of accuracy but as a means to refine estimates (van Oort 2005; Foody, 2009) and to provide the matrix as part of the accuracy statement. The results show that with known ground data quality, the real land cover change values may be derived by simple algebraic means.

The basic latent class modelling approach represented by equation 15 may also be used when errors are independent. The model based upon the use of equation 23 was used to derive land cover change estimates using just the outputs simulated for the remote sensing change detection classifiers in scenarios B, I, J and $\mathrm{K}$. These data sets had been generated in a way to ensure independent errors, a property that was confirmed by the log-odds ratio check analysis (Table 3). The outputs of the four classifiers were used to construct a $2^{4}$ cross-tabulation that illustrated the pattern of class labels derived from the classifications to drive the analysis (Table 4). The model was found to fit the data closely $\left(L^{2}=5.03, \mathrm{df}=6\right)$, and the producer's accuracy (sensitivity) and prevalence of change estimated from the results of the analysis are shown in Table 5. Note that the values shown in Table 4 were derived without any reference to ground reference data and are close to the real values, with the largest difference in producer's accuracy being $3.2 \%$ for the classifier in scenario B. 
Finally, the data in scenario $\mathrm{K}$ were replaced by those in scenario L, which was highly correlated with the data in scenario $\mathrm{J}$ to illustrate the situation in which the conditional independence cannot be assumed. Again a $2^{4}$ cross-classification table was formed for input to the analysis (Table 6) and estimates of the sensitivity of the classifications and change prevalence derived from the model. For illustrative purposes the model based on the use of equation 23 , which is based on conditional independence (i.e. a standard model which fails to recognise the known dependence between the classification outputs for scenarios $\mathbf{J}$ and $\mathrm{L}$ ), was applied to the data. The results of this analysis were found to fit the data poorly $\left(L^{2}=159.59, \mathrm{df}=4\right)$ and the derived estimates differed greatly from reality (Table 7). Repeating the analysis but using the model based on equation 24 , which allowed for the known conditional dependence between the data from scenarios $\mathrm{J}$ and $\mathrm{L}$ (Table 2), yielded a model that fitted more closely with the data $\left(L^{2}=81.09, \mathrm{df}=4\right)$. Although this model fitted the data more closely and was not a good fit the estimates of prevalence and accuracy derived from it were reasonably close to reality (Table 8). The results, together with those reported elsewhere (e.g. Torrance-Rynard and Walter, 1998), indicate that sometimes it is possible to derive accurate estimates from non-ideal latent class models. For example, the largest difference between the predicted and actual producer's accuracy was 3.0\% for the classification from scenario B. It was apparent, however, that there was evidence for conditional dependence between the data from scenarios B and L (Table 9). This dependence between the data in scenarios B and L (Table 2) was unplanned and reinforces the need to test for conditional independence and use an appropriate model of the dependence structure with a latent class analysis. The 
dependency arose from the approach used to derive the data for scenario L from that in scenario J; the 150 cases for which the class label were changed were not selected randomly but from a data set ordered by perfect ground data which was in turn linked directly to the data for scenario B. Adjusting the model component in equation 24 to allow for a more complex dependence structure, including the dependence of B and L, and repeating the analysis resulted in a model that provided even closer fit to the data $\left(L^{2}=37.71, \mathrm{df}=2\right)$. Additionally, the estimates of accuracy and prevalence derived were close to reality (Table 10), although not necessarily closer than those derived from the earlier model, but the analysis was free from significant problems of conditional dependence (Table 11). It is worth stressing again, that the estimates of accuracy and change extent were derived without use of ground reference data.

Although the latent class modelling is more complex than the simple algebraic approach for the correction of the impacts of an imperfect reference it does appear to have considerable potential. Additionally, the approach can sometimes be implemented as a log-linear model form, a type of analysis with which there is some familiarity in the remote sensing community. There are, however, some important concerns with latent class modelling. There are, for example, important concerns linked to the identifiability of the latent class model (Rindskopf and Rindskopf, 1986; Uebersax and Grove, 1990) and especially the dependence model used (Torrance-Rynard and Walter, 1997; Albert and Dodd, 2004; Albert et al., 2001 Pepe and Janes, 2007). Indeed it must be explicitly recognised that the latent class modelling approach is not a panacea for ground reference data problems. Critically, it is not suggested that the approach can free researchers from 
the need for ground reference data or from the potential to make erroneous estimation. The latent class modelling approach may sometimes offer an ability to constructively address ground reference data problems but its use does involve strong assumptions, especially if conditional dependence occurs, and so must be used with care. This paper has sought to show that ground reference data error may have substantial negative impacts on the remote sensing of land cover change but that sometimes it is possible to quantify these impacts and even implement corrective actions to reduce or remove them. Further work is needed to develop the latter, with the potential and limitations of latent class modelling, in particular, deserving greater attention in remote sensing.

\section{Conclusions}

The basis of change detection by remote sensing is very simple. In many applications the key information on change detection accuracy and amount or extent of change may be derived from a binary change detection matrix that is no more than a cross-tabulation of the labels contained the ground reference data set against those in derived from the remote sensing change detection analysis. There are, however, many sources of error in studies of land cover change and this paper focused on just one issue, the problems arising from the use of an imperfect ground reference data set.

The use of an imperfect ground reference data set may introduce substantial bias into the estimates of land cover change variables. While the accuracy of ground reference data has been known to be a concern for a long time its impacts have previously been poorly 
understood and, aside from a few studies such as Hagen (2003), very little action taken to address negative effects. However, the results have shown that even small errors in the ground reference data set may introduce large bias into the derived estimates and so it is extremely unsafe to assume an error-free or gold standard reference data set. Fortunately, there are sometimes ways to address the problems caused by ground reference data error. Some methods that may be used were illustrated in this paper for a range of situations. It was stressed that the nature of the errors, especially in relation to the underlying assumptions of the techniques, has important implications that should not be ignored as deviation from the assumed condition can have a major negative effect on the analysis. While this article has focused on the potential of techniques such as latent class modelling in remote sensing further investigation is required. The latter should explore the limitations of latent class modelling and its suitability for use in typical remote sensing contexts. None-the-less, the potential to reduce or even remove the bias caused by ground reference data error has been indicated and this may help the effective use of remote sensing as a source of information on land cover change. The techniques discussed are, of course, also applicable beyond studies of change, notably to other binary classification problems.

In summary, the four main conclusions of this paper are:

1. Ground reference data error can be a source of considerable error and misinterpretation in studies of land cover change. It can, for example, lead to over- or under-estimation of some measures of accuracy and mis-estimation of the extent of change. The specific effects arising from the use of an imperfect ground 
reference data set vary with the nature of the errors it contains (e.g. if correlated or not with the errors in the remotely sensed data).

2. The magnitude of the producer's accuracy (sensitivity) can, contrary to widespread belief in some communities, vary as a function of the prevalence of change if an imperfect ground reference data set is used. The use of an imperfect reference data set also impacts on the user's and overall classification accuracy.

3. It is sometimes possible to reduce or even remove the effects of ground data error. Moreover, it is sometimes possible to derive accurate estimates of change detection accuracy and extent without ground data. Note that the approach founded on latent class analysis is based on a model and the satisfaction of the model assumptions is critical in its use.

4. Ground data error and its impacts should be considered in the interpretation of studies of land cover change.

\section{Acknowledgments}

This work has benefited from feedback received from conference presentations and referees as it evolved over the last three years. I am grateful for software resources used that were freely available on the internet, notably the LEM software for the latent class analyses and the CONDEP software for the log-odds ratio check analyses. I am also very grateful to the three referees for their very helpful comments on the original manuscript, especially one referee who provided a remarkably thorough and constructive set of review comments, which greatly enhanced the article. 


\section{References}

Achard, F., Eva, H. D., Stibig, H-J., Mayaux, P., Gallego, J., Richards, T. and Malingreau, J-P. (2002) Determination of deforestation rates of the world's humid tropical forests, Science, 297, 999-1002.

Albert, P. S., and Dodd, L. E. (2004) A cautionary note on the robustness of latent class models for estimating diagnostic error without a gold standard, Biometrics, 60, 427-435.

Albert, P. S., McShane, L. M. and Shih, J. H. (2001) Latent class modeling approaches for assessing diagnostic error without a gold standard: With applications to p53 immunohistochemical assays in bladder tumors, Biometrics, 57, 610-619.

Alonzo, T. A., Pepe, M. S. and Moskowitz, C. S. (2002) Sample size calculations for comparative studies of medical tests for detecting presence of disease, Statistics in Medicine, 21, 835-852.

Baraldi, A., Bruzzone, L. and Blonda, P. (2005) Quality assessment of classification and cluster maps without ground truth knowledge, IEEE Transactions on Geoscience and Remote Sensing, 43, 857-873. 
Becker, M. P. and Yang, I. (1998) Latent class marginal models for cross-classifications of counts, Sociological Methodology, 28, 293-325.

Bradley, B. A. (2009) Accuracy assessment of mixed land cover using a GIS-designed sampling scheme, International Journal of Remote Sensing, 30, 3515-3529.

Branscum, A. J., Gardner, I. A. and Johnson, W. O. (2005) Estimation of diagnostic-test sensitivity and specificity through Bayesian modeling, Preventive Veterinary Medicine, $68,145-163$.

Brannstrom, C. and Filippi, A. M. (2008) Remote classification of Cerrado (Savanna) and agricultural land covers in northeastern Brazil, Geocarto International, 23, 109-134.

Brannstrom, C., Jepson, W., Filippi, A. M., Redo, D., Xu, Z. and Ganesh, S. (2008) Land change in the Brazilian Savanna (Cerrado), 1986-2002: comparative analysis and implications for land-use policy, Land Use Policy, 25, 579-595.

Broich, M., Stehman, S. V., Hansen, M. C., Potapov, P. and Shimabukuro, Y. E. (2009) A comparison of sampling designs for estimating deforestation from Landsat imagery: a case study from the Brazilian Legal Amazon, Remote Sensing of Environment, in press (doi:10.1016/j.rse.2009.07.011) 
Bruzzone, L. and Marconcini, M. (2009) Toward the automatic updating of land-cover maps by a domain-adaptation SVM classifier and a circular validation strategy, IEEE Transactions on Geoscience and Remote Sensing, 47, 1108-1122.

Bruzzone, L. and Persello, C. (2009) A novel context-sensitive semisupervised SVM classifier robust to mislabeled training samples, IEEE Transactions on Geoscience and Remote Sensing, 47, 2142-2154.

Bruzzone, L., Cossu, R. and Vernazza, G. (2004) Detection of land-cover transitions by combining multidate classifiers, Pattern Recognition Letters, 25, 1491-1500.

Buck, A. A. and Gart, J. J. (1966) Comparison of a screening test and a reference test in epidemiologic studies. I Indices of agreement and their relationship to prevalence, American Journal of Epidemiology, 83, 586-592.

Carlotto, M. J. (2009) Effect of errors in ground truth on classification accuracy, International Journal of Remote Sensing, 30, in press.

Cihlar, J. (2000) Land cover mapping of large areas from satellites: status and research priorities, International Journal of Remote Sensing, 21, 1093-1114. 
Chen, J., Gong, P., He, C., Pu, R. and Shi, P. (2003) Land-use/land-cover change detection using improved change-vector analysis, Photogrammetric Engineering and Remote Sensing, 69, 369-379.

Comber, A., Fisher, P. and Wadsworth, R. (2005) What is land cover? Environment and Planning B, 32, 199-209.

Congalton, R. G. (1988) Using spatial auto-correlation analysis to explore the errors in maps generated from remotely sensed data, Photogrammetric Engineering and Remote Sensing, 54, 587-592.

Congalton, R. G. (1991) A review of assessing the accuracy of classifications of remotely sensed data, Remote Sensing of Environment, 37, 35-46.

Congalton, R. G. and Green, K. (2009) Assessing the Accuracy of Remotely Sensed Data: Principles and Practices, second edition, Boca Raton, Lewis Publishers

Dale, V. H. (1997) The relationship between land-use change and climate change, Ecological Applications, 7, 753-769.

DeFries, R. S., Houghton, R. A., Hansen, M. C., Field, C. B., Skole, D. and Townshend, J. (2002) Carbon emissions from tropical deforestation and regrowth based on satellite observations for the 1980s and 1990s, Proceedings of the National Academy of Sciences USA, 99, 14256-14261. 
Duro, D., Coops, N. C., Wulder, M. A. and Han, T. (2007) Development of a large area biodiversity monitoring system driven by remote sensing, Progress in Physical Geography, 31, 235-260.

Enøe, C., Georgiadis, M. P. and Johnson, W. O. (2000) Estimation of sensitivity and specificity of diagnostic tests and disease prevalence when the true disease state is unknown, Preventive Veterinary Medicine, 45, 61-81.

Enøe, C., Anderson, S., Sørensen, V. and Willeberg, P. (2001) Estimation of sensitivity, specicity and predictive values of two serologic tests for the detection of antibodies against Actinobacillus pleuropneumoniae serotype 2 in the absence of a reference test (gold standard), Preventive Veterinary Medicine, 51, 227-243.

Engels, E. A., Sinclair, M. D., Biggar, R. J., Whitby, D., Ebbesen, P., Goedert, J. J. and Gastwirth, J. L. (2000) Latent class analysis of human herpesvirus 8 assay performance and infection prevalence in sub-saharan Africa and Malta, International Journal of Cancer, 88, 1003-1008.

Eshleman, K.N. (2004) Hydrological consequences of land use change: a review of the state-of-science. In Ecosystems and Land Use Change (R.S. DeFries, G.P. Asner, and R.A. Houghton, Eds.), pp. 13-29, Geophysical Monograph Series 153, American Geophysical Union, Washington, DC. 
Espeland, M. A. and Handelman, S. L. (1989) Using latent class models to characterize and assess relative error in discrete measurements, Biometrics, 45, 587-599.

Fielding, A. H. and Bell, J. F. (1997) A review of methods for the assessment of prediction errors in conservation presence/absence models, Environmental Conservation, 24, 38-49.

Foody, G. M. (2002) Status of land cover classification accuracy assessment, Remote Sensing of Environment, 80, 185-201.

Foody, G. M. (2008) Harshness in image classification accuracy assessment, International Journal of Remote Sensing, 29, 3137-3158.

Foody, G. M. (2009) The impact of imperfect ground reference data on the accuracy of land cover change estimation, International Journal of Remote Sensing, 30, 3275-3281.

Foulds, S. A. and Macklin, M. G. (2006) Holocene land-use change and its impact on river basin dynamics in Great Britain and Ireland, Progress in Physical Geography, 30, $589-604$.

Gallego, F. J. (2004) Remote sensing and land cover area estimation, International Journal of Remote Sensing, 25, 3019-3047. 
Gardner, I. A., Stryhn, H., Lind, P. and Collins, M. T. (2000) Conditional dependence between tests affects the diagnosis and surveillance of animal diseases, Preventive Veterinary Medicine, 45, 107-122.

Garrett, E. S. and Zeger, S. L. (2000) Latent class model diagnosis, Biometrics, 56, 10551067.

Gart, J. J. and Buck, A. A. (1966) Comparison of a screening test and a reference test in epidemiologic studies: II a probabilistic model for the comparison of diagnostic tests, American Journal of Epidemiology, 83, 593-602.

Georgiadis, M., Johnson, W. O., Garner, I. A. and Singh, R. (2003) Correlation-adjusted estimation of sensitivity and specificity of two diagnostic tests, Applied Statistics, 52, 6378.

Gillespie, T. W., Foody, G. M., Rocchini, D., Giorgi, A. P. and Saatchi, S. (2008) Measuring and modelling biodiversity from space, Progress in Physical Geography, 32, 203-221.

Goetghebeur, E., Liinev, J., Boelaert, M and van der Stuyft, P. (2000) Diagnostic test analyses in search of their gold standard: latent class analysis with random effects, Statistical Methods in Medical Research, 9, 231-248. 
Hagen, A (2003) Fuzzy set approach to assessing similarity of categorical maps, International Journal of Geographical Information Science, 17, 235-249.

Hawkins, D. M., Garrett, J. A. and Stephenson, B. (2001) Some issues in resolution of diagnostic tests using an imperfect gold standard, Statistics in Medicine, 20, 1987-2001.

Herold, M., Woodcock, C. E., di Gregorio, A., Mayaux, P., Belward, A. S., Latham, J. and Schmullius, C. (2006) A joint initiative for harmonization of land cover data sets, IEEE Transactions on Geoscience and Remote Sensing, 44, 1719-1727.

Herold, M., Mayaux, P., Woodcock, C. E., Baccini, A. and Schmullius, C. (2008) Some challenges in global land cover mapping: An assessment of agreement and accuracy in existing $1 \mathrm{~km}$ datasets, Remote Sensing of Environment, 112, 2538-2556.

Hui, S. L. and Zhou, X. H. (1998) Evaluation of diagnostic tests without gold standards, Statistical Methods in Medical Research, 7, 354-370.

Jepson, W. (2005) A disappearing biome? Reconsidering land-cover change in the Brazilian savanna, The Geographical Journal, 171, 99-111.

Johnson, E. W. and Ross J. (2008) Quantifying error in aerial survey data, Australian Forestry, 71, 216-222. 
Jones, D. A., Hansen, A. J., Bly, K., Doherty, K., Verschuyl, J. P., Paugh, J. I., Carle, R. and Story, S. J. (2009) Monitoring land use and cover around parks: a conceptual approach, Remote Sensing of Environment, 113, 1346-1356.

Justice, C., Belward, A., Morisette, J., Lewis, P., Privette, J. and Baret, F. (2000) Developments in the 'validation' of satellite sensor products for the study of the land surface, International Journal of Remote Sensing, 21, 3383-3390.

Kennedy, R. E., Townsend, P. A., Gross, J. E., Cohen, W. B., Bolstad, P., Wang, Y. Q. and Adams, P. (2009) Remote sensing change detection tools for natural resource managers: understanding concepts and tradeoffs in the design of landscape monitoring projects, Remote Sensing of Environment, 113, 1382-1396.

Kintisch, E. (2007) Improved monitoring of rainforests helps pierce haze of deforestation, Science, 316, 536-537.

Khorram, S. (Ed), (1999) Accuracy Assessment of Remote Sensing-Derived Change Detection, (Bethesda, MD: American Society for Photogrammetry and Remote Sensing).

Liu, C., White, M. and Newell, G. (2009) Measuring the accuracy of species distribution models: a review, Proceedings $18^{\text {th }}$ World IMACs/MODSIM Congress, Cairns, Australia, 4241-4247. 
Liu, H. and Zhou, Q. (2004) Accuracy analysis of remote sensing change detection by rule-based rationality evaluation with post-classification comparison, International Journal of Remote Sensing, 25, 1037-1050.

Lunetta, R. S., Knight, J. F., Ediriwickrema, J., Lyon, J. G. and Worthy, L. D. (2006) Land-cover change detection using multi-temporal MODIS NDVI data, Remote Sensing of Environment, 105, 142-154.

Lu, D., Batistella, M., Moran, E. and de Miranda, E. E. (2008) A comparative study of Landsat TM and SPOT HRG images for vegetation classification in the Brazilian Amazon, Photogrammetric Engineering and Remote Sensing, 74, 311-321.

Magidson, J. and Vermunt, J. K. (2004) Latent class models, In Kaplan, D (editor) The SAGE Handbook of Quantitative Methodology for the Social Sciences, Sage, Thousand Oaks, 175-198.

Mann, S. and Rothley, K. D. (2006) Sensitivity of Landsat/IKONOS accuracy comparison to errors in photointerpreted reference data and variations in test point sets, International Journal of Remote Sensing, 27, 5027-5036.

Mannel, S., Price, M. and Hua, D. (2006) A method to obtain large quantities of reference data, International Journal of Remote Sensing, 27, 623-627. 
McAlpine, C. A. Syktus, J., Ryan J. G., Deo, R. C., McKeon, G. M., McGowan, H. A., and Phinn, S. R. (2009) A continent under stress: interactions, feedbacks and risks associated with impact of modified land cover on Australia's climate, Global Change Biology, 15, 2206-2223.

Messam, L. L. McV., Branscum, A. J., Collins, M. T. and Gardner, I. A. (2008) Frequentist and Bayesian approaches to prevalence estimation using examples from Johne's disease, Animal Health Research Reviews, 9, 1-23.

Miller, W. C. (1998) Can we do better than discrepant analysis for new diagnostic test evaluation, Clinical Infectious Diseases, 27, 1186-1193.

Mundia, C. N. and Aniya, M. (2005) Analysis of land use/cover changes and urban expansion of Nairobi city using remote sensing and GIS, International Journal of Remote Sensing, 26, 2831-2849.

Patil, G. P. and Taillie, C. (2003) Modeling and interpreting the accuracy assessment error matrix for a doubly classified map, Environmental and Ecological Statistics, 10, $357-373$.

Pepe, M. S., and Janes, H. (2007) Insights into latent class analysis of diagnostic test performance, Biostatistics, 8, 474-484. 
Pontius, R. G. and Lippitt, C. D. (2006) Can error explain map differences over time? Cartography and Geographic Information Science, 33, 159-171.

Pontius, R. G. and Petrova, S. H. (2010) Assessing a predictive model of land change using uncertain data, Environmental Modelling and Software, 25, 299-309.

Potere, D., Schneider, A., Angel, S. and Civco, D. A. (2009) Mapping urban areas on a global scale: which of the eight maps now available is more accurate? International Journal of Remote Sensing, 30, (in press).

Powell, R. L., Matzke, N., de Souza, C., Clark, M., Numata, I., Hess, L. L. and Roberts, D. A. (2004) Sources of error in accuracy assessment of thematic land-cover maps in the Brazilian Amazon, Remote Sensing of Environment, 90, 221-234.

Qu, Y. and Hadgu, A. (1998) A model for evaluating sensitivity and specificity for correlated diagnostic tests in efficacy studies with an imperfect reference test, Journal of the American Statistical Association, 93, 920-928.

Qu, Y., Tan, M. and Kutner, M. H. (1996) Random effects models in latent class analysis for evaluating accuracy of diagnostic tests, Biometrics, 52, 797-810. 
Rahme, E. and Joseph, L. (1998) Estimating the prevalence of a rare disease: adjusted maximum likelihood, The Statistician, 47, 149-158.

Rindfuss, R. R., Walsh, S. J., Turner Ii, B. L., Fox, J. and Mishra, V. (2004) Developing a science of land change: challenges and methodological issues, Proceedings of the National Academy of Sciences USA, 101, 13976-13981.

Rindskopf, D. (2002) The use of latent class analysis in medical diagnosis, Proceedings of the Annual Meeting of the American Statistical Association, American Statistical Association, Alexandria VA, 2912-2916.

Rindskopf, D. and Rindskopf, W. (1986) The value of latent class analysis in medical diagnosis, Statistics in Medicine, 5, 21-27.

Rogan, J., Miller, J., Stow, D., Franklin, J., Levien, L. and Fischer, C. (2003) Land-cover change monitoring with classification trees using Landsat TM and ancillary data, Photogrammetric Engineering and Remote Sensing, 69, 793-804.

Rogan, W. J. and Gladen, B. (1978) Estimating prevalence from the results of a screening test, American Journal of Epidemiology, 107, 71-76. 
See, L .M.and Fritz, S. (2006) A method to compare and improve land cover datasets: Application to the GLC-2000 and MODIS land cover products, IEEE Transactions on Geoscience and Remote Sensing, 44, 1740-1746.

Shao, G. and $\mathrm{Wu}$, J. (2008) On the accuracy of landscape pattern analysis using remote sensing data, Landscape Ecology, 23, 505-511.

Simon, D. and Boring, J. R. (1990) Sensitivity, Specicity and predictive value, In H. K. Walker, W. D. Hall and J. W. Hurst (editors) Clinical Methods. The History, Physical and Laboratory Examinations, third edition, Butterworths, 49-54.

Skole, D. and Tucker, C. (1993) Tropical deforestation and habitat fragmentation in the Amazon - satellite data from 1978 to 1988, Science, 260, 1905-1910.

Staquet, M., Rozencweig, M., Lee, Y. J. and Muggia, F. M. (1981) Methodology for the assessment of new dichotomous diagnostic tests, Journal of Chronic Diseases, 34, 599610.

Stehman, S. V. (2005) Comparing estimators of gross change derived from complete coverage mapping versus statistical sampling of remotely sensed data. Remote Sensing of Environment, 96, 466-474. 
Stehman, S. V. (2009) Sampling designs for accuracy assessment of land cover, International Journal of Remote Sensing, 30, (in press).

Strahler, A. H., Boschetti, L., Foody, G. M., Friedl, M. A., Hansen, M. C., Herold, M., Mayaux, P., Morisette, J. T., Stehman, S. V. and Woodcock, C. E. (2006) Global Land Cover Validation: Recommendations for Evaluation and Accuracy Assessment of Global Land Cover Maps, Technical Report, Joint Research Centre, Ispra, EUR 22156 EN, 48pp.

Thompson, I. D., Maher, S. C., Rouillard, D. P., Fryxell, J. M. and Baker, J. A. (2007) Accuracy of forest inventory mapping, some implications for boreal forest management, Forest Ecology and Management, 252, 208-221.

Torrance-Rynard, V. L. and Walter, S. D. (1997) Effects of dependent errors in the assessment of diagnostic test performance, Statistics in Medicine, 16, 2157-2175.

Townshend, J. R. G. (1992) Land cover, International Journal of Remote Sensing, 13, $1319-1328$.

Treitz, P. and Rogan, J. (2004) Remote-sensing for mapping and monitoring land-cover and land-use change - an introduction, Progress in Planning, 61, 269-279.

Tucker, C. J., Townshend, J. R. G. and Goff, T. E. (1985) African land-cover classification using satellite data, Science, 227, 369-375. 
Turner II, B. L., Lambin, E. F. and Reenberg, A. (2007) The emergence of land change science for global environmental change and sustainability, Proceedings of the National Academy of Sciences of the United States of America, 104, 20666-20671.

Uebersax, J. S. and Grove, W. M. (1990) Latent class analysis of diagnostic agreement, Statistics in Medicine, 9, 559-572.

Vacek, P. M. (1985) The effect of conditional dependence on the evaluation of diagnostic tests, Biometrics, 41, 959-968.

Valenstein, P. N. (1990) Evaluating diagnostic tests with imperfect standards, American Journal of Clinical Pathology, 93, 252-258.

van Oort, P. A. J. (2005) Improving land cover change estimates by accounting for classification errors, International Journal of Remote Sensing, 26, 3009-3024.

van Oort, P. A. J. (2007) Interpreting the change detection error matrix, Remote Sensing of Environment, 108, 1-8.

Verbyla, D. L. and Boles, S. H. (2000) Bias in land cover change estimates dues to misregistration, International Journal of Remote Sensing, 21, 3553-3560. 
Vermunt, J. K. (1997) Log-linear Models for Event Histories, Sage, Thousand Oaks.

Vitousek, P. M. (1994) Beyond global warming - ecology and global change, Ecology, $75,1861-1876$.

Weng, Q. H. (2002) Land use change analysis in the Zhujiang delta of China using satellite remote sensing, GIS and stochastic modelling, Journal of Environmental Management, 64, 273-284.

Wilkinson, G. G. (1996) Classification algorithms - where next? Soft Computing in Remote Sensing Data Analysis, (E. Binaghi, P. A. Brivio and A. Rampini, editors), World Scientific, Singapore, pp. 93-99.

Wilkinson, G. G. (2005) Results and implications of a study of fifteen years of satellite image classification experiments, IEEE Transactions on Geoscience and Remote Sensing, $43,433-440$.

Woodcock, C. E., Macomber, S. A., Pax-Lenney, M. and Cohen, W. B. (2001) Monitoring large areas for forest change using Landsat: generalisation across space, time and Landsat sensors, Remote Sensing of Environment, 78, 194-203.

Xu, Y. G., Dickson, B. G., Hampton, H. M., Sisk, T. D., Palumbo, J. A. and Prather, J. W. (2009) Effects of Mismatches of Scale and Location between Predictor and Response 
Variables on Forest Structure Mapping, Photogrammetric Engineering and Remote Sensing, 75, 313-322.

Yang, I. and Becker, M. P. (1997) Latent variable modelling of diagnostic accuracy, Biometrics, 53, 948-958.

Yang, X. and Liu, Z. (2005) Using satellite imagery and GIS for land-use and land-cover change mapping in an estuarine watershed, International Journal of Remote Sensing, 26, $5275-5296$.

Yang, X. and Lo, C. P. (2002) Using a time series of satellite imagery to detect land use and land cover changes in the Atlanta, Georgia metropolitan area, International Journal of Remote Sensing, 23, 1775-1798. 
Table 1. Five scenarios used to explore the impacts of ground reference data error on the accuracy of change detection and change extent estimation. Note that in each of these scenarios the errors in the ground and remotely sensed data sets were independent of each other.

\begin{tabular}{|c|c|c|c|}
\hline \multirow[b]{2}{*}{ Scenario } & \multicolumn{2}{|c|}{ Classification accuracy $(\%)$} & \multirow{2}{*}{$\begin{array}{c}\text { True } \\
\text { prevalence }(\%)\end{array}$} \\
\hline & Remote sensing & Ground data & \\
\hline A & $S_{1}=S_{2}=90.0$ & $S_{1}^{\prime}=S_{2}^{\prime}=95.0$ & 20.0 \\
\hline B & $S_{1}=S_{2}=80.0$ & $S_{1}^{\prime}=S_{2}^{\prime}=90.0$ & 20.0 \\
\hline $\mathrm{C}$ & $S_{1}=S_{2}=65.0$ & $S_{1}^{\prime}=S_{2}^{\prime}=70.0$ & 20.0 \\
\hline D & $S_{1}=S_{2}=80.0$ & $S_{1}^{\prime}=80.0 \quad S_{2}^{\prime}=100.0$ & 20.0 \\
\hline $\mathrm{E}$ & $S_{1}=S_{2}=80.0$ & $S_{1}^{\prime}=S_{2}^{\prime}=90.0$ & 5.0 \\
\hline
\end{tabular}


Table 2. Conditional correlations $\rho_{1}\left(\rho_{0}\right)$ between pairs of data sets derived for scenarios $\mathrm{B}, \mathrm{I}, \mathrm{J}, \mathrm{K}$, and L.

\begin{tabular}{lccccc} 
& B & I & J & K & L \\
\hline B & - & $0.051(0.021)$ & $0.033(-0.028)$ & $-0.085(-0.042)$ & $0.492(0.008)$ \\
I & & - & $-0.104(0.087)$ & $-0.009(-0.013)$ & $0.023(0.106)$ \\
J & & & - & $-0.043(0.142)$ & $0.398(0.722)$ \\
K & & & & - & $-0.070(0.019)$
\end{tabular}


Table 3. Log-odds ratio check output for the latent class analysis based on variables defined in scenarios B, I, J and K for an analysis using equation 23 .

\begin{tabular}{|c|c|c|c|c|c|}
\hline \multicolumn{2}{|c|}{$\begin{array}{l}\text { Variables } \\
\text { compared }\end{array}$} & $\begin{array}{l}\text { Expected } \\
\text { log odds } \\
\text { ratio }\end{array}$ & s.e. & $\begin{array}{l}\text { Observed } \\
\text { log odds } \\
\text { ratio }\end{array}$ & $\begin{array}{l}z^{-} \\
\text {value }\end{array}$ \\
\hline & & --- & ----- & ----- & ------ \\
\hline B & I & 1.85 & 0.157 & 1.84 & -0.05 \\
\hline B & $\mathrm{J}$ & 1.39 & 0.149 & 1.37 & -0.11 \\
\hline B & $\mathrm{K}$ & 0.62 & 0.138 & 0.48 & -1.03 \\
\hline I & $\mathrm{J}$ & 2.26 & 0.164 & 2.25 & -0.04 \\
\hline I & K & 1.02 & 0.148 & 1.02 & 0.03 \\
\hline $\mathrm{J}$ & $\mathrm{K}$ & 0.77 & 0.143 & 0.84 & 0.51 \\
\hline
\end{tabular}


Table 4 . The $2^{4}$ cross-tabulation of outputs $(b, i, j, k)$ from the remote sensing classifiers used in scenarios B, I, J and K. The table shows the number of times a particular combination of labels was derived (e.g. all four classifiers predicted change for 77 of the 1000 cases).

\begin{tabular}{|c|c|}
\hline$\underline{b i j k}$ & $n$ \\
\hline 1111 & 77 \\
\hline 1110 & 46 \\
\hline 1101 & 25 \\
\hline 1100 & 16 \\
\hline 1011 & 14 \\
\hline 1010 & 16 \\
\hline 1001 & 31 \\
\hline 1000 & 95 \\
\hline 01111 & 25 \\
\hline 0110 & 18 \\
\hline $\begin{array}{llll}0 & 1 & 0 & 1\end{array}$ & 20 \\
\hline 0100 & 34 \\
\hline $\begin{array}{llll}0 & 0 & 1 & 1\end{array}$ & 32 \\
\hline 0010 & 53 \\
\hline 0001 & 157 \\
\hline 0000 & 341 \\
\hline
\end{tabular}


Table 5. Results derived form the latent class analysis based on equation 23; note the estimates were derived without the use of ground reference data. The actual values were known for simulated data and may be derived from the confusion matrices shown in Figures 2 and 5.

\begin{tabular}{lll} 
Property & Estimate $(\%)$ & Actual $(\%)$ \\
\hline Prevalence & 21.6 & 20.0 \\
Classifier B, $S_{1}$ & 76.8 & 80.0 \\
Classifier I, $S_{1}$ & 91.3 & 90.5 \\
Classifier J, $S_{1}$ & 79.6 & 82.5 \\
Classifier K, $S_{1}$ & 64.3 & 67.0
\end{tabular}


Table 6 . The $2^{4}$ cross-tabulation of outputs $(b, i, j, l)$ from the remote sensing classifiers used in scenarios B, I, $\mathrm{J}$ and $\mathrm{L}$.

\begin{tabular}{|c|c|}
\hline $\begin{array}{l}b i j l \\
\end{array}$ & $r$ \\
\hline 1111 & 115 \\
\hline 1110 & 8 \\
\hline 1101 & 4 \\
\hline 1100 & 37 \\
\hline 1011 & 28 \\
\hline 1010 & 2 \\
\hline 1001 & 16 \\
\hline 1000 & 110 \\
\hline $0 \begin{array}{llll}0 & 1 & 1 & 1\end{array}$ & 14 \\
\hline 0110 & 29 \\
\hline 0101 & 17 \\
\hline 0100 & 37 \\
\hline 00011 & 68 \\
\hline 0010 & 17 \\
\hline 0001 & 57 \\
\hline 0000 & 44 \\
\hline
\end{tabular}


Table 7. Results derived from the latent class analysis based on equation 23 to the data from scenarios B, I, J and L; note the estimates were derived without the use of ground reference data. The actual values were known for simulated data and may be derived from the confusion matrices shown in Figures 2 and 5.

\begin{tabular}{lll} 
Property & Estimate $(\%)$ & Actual $(\%)$ \\
\hline Prevalence & 28.0 & 20.0 \\
Classifier B, $S_{1}$ & 56.8 & 80.0 \\
Classifier I, $S_{1}$ & 61.6 & 90.5 \\
Classifier J, $S_{1}$ & 94.4 & 82.5 \\
Classifier L, $S_{1}$ & 83.7 & 66.5
\end{tabular}


Table 8. Results derived from the latent class analysis based on equation 24 to the data from scenarios B, I, J and L; note the estimates were derived without the use of ground reference data. The actual values were known for simulated data and may be derived from the confusion matrices shown in Figures 2 and 5.

\begin{tabular}{lll} 
Property & Estimate $(\%)$ & Actual $(\%)$ \\
\hline Prevalence & 21.2 & 20.0 \\
Classifier B, $S_{1}$ & 77.0 & 80.0 \\
Classifier I, $S_{1}$ & 92.4 & 90.5 \\
Classifier J, $S_{1}$ & 79.8 & 82.5 \\
Classifier L, $S_{1}$ & 67.8 & 66.5
\end{tabular}


Table 9. Log-odds ratio check output for the latent class analysis based on variables defined in scenarios B, I, J and L for an analysis using equation 24 , with dependence between $\mathbf{J}$ and $\mathrm{L}$.

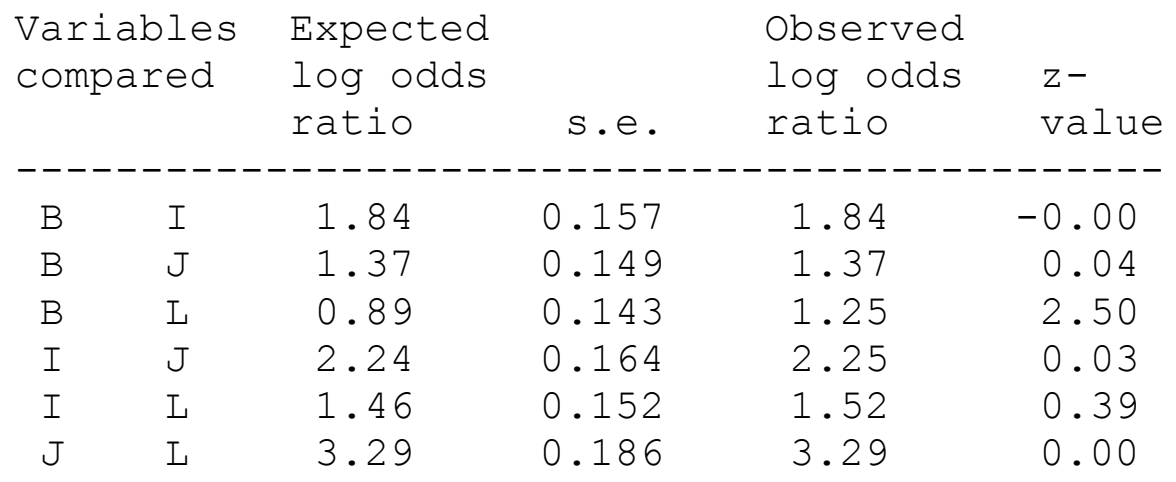


Table 10. Results derived from the latent class analysis based to the data from scenarios $\mathrm{B}, \mathrm{I}, \mathrm{J}$ and $\mathrm{L}$ allowing for conditional dependence between $\mathrm{B}$ and $\mathrm{L}$ and $\mathrm{J}$ and $\mathrm{L}$; note the estimates were derived without the use of ground reference data. The actual values were known for simulated data and may be derived from the confusion matrices shown in Figures 2 and 5.

\begin{tabular}{lll} 
Property & Estimate $(\%)$ & Actual $(\%)$ \\
\hline Prevalence & 20.7 & 20.0 \\
Classifier B, $S_{1}$ & 75.3 & 80.0 \\
Classifier I, $S_{1}$ & 91.9 & 90.5 \\
Classifier J, $S_{1}$ & 82.0 & 82.5 \\
Classifier L, $S_{1}$ & 65.4 & 66.5
\end{tabular}


Table 11. Log-odds ratio check output for the latent class analysis based on variables defined in scenarios B, I, J and L for an analysis using equation 24 , with dependence between $\mathrm{J}$ and $\mathrm{L}$ and $\mathrm{B}$ and $\mathrm{L}$.

\begin{tabular}{|c|c|c|c|c|c|}
\hline \multicolumn{2}{|c|}{$\begin{array}{l}\text { Variables } \\
\text { compared }\end{array}$} & $\begin{array}{l}\text { Expected } \\
\text { log odds } \\
\text { ratio }\end{array}$ & s.e. & $\begin{array}{l}\text { Observed } \\
\text { log odds } \\
\text { ratio }\end{array}$ & $\begin{array}{l}z^{-} \\
\text {value }\end{array}$ \\
\hline & & & ----- & ----- & -----1 \\
\hline B & I & 1.70 & 0.155 & 1.84 & 0.95 \\
\hline B & $\mathrm{J}$ & 1.56 & 0.150 & 1.37 & -1.21 \\
\hline B & $\mathrm{L}$ & 1.25 & 0.144 & 1.25 & 0.00 \\
\hline I & $\mathrm{J}$ & 2.25 & 0.164 & 2.25 & 0.00 \\
\hline I & $\mathrm{L}$ & 1.31 & 0.151 & 1.52 & 1.39 \\
\hline $\mathrm{J}$ & $\mathrm{L}$ & 3.29 & 0.186 & 3.29 & 0.00 \\
\hline
\end{tabular}




\section{Figure captions}

Figure 1. The binary confusion matrix. Each case is allocated to one of the elements of the $2 \times 2$ matrix (highlighted in grey) and a suite of statistics may be derived from the matrix entries relative to the marginal values. All other matrices presented in this paper are formatted in the style shown here.

Figure 2.The observed and real confusion matrices based on imperfect and perfect ground reference data respectively together with associated derived values for scenarios A-E. Note that in each of the scenarios depicted the data were generated in a fashion to ensure that the assumption of conditional independence was satisfied.

Figure 3. The confusion matrices and derived values illustrating impacts of correlated errors. (a) the real matrix, (b) scenario $\mathrm{F}$, matrix derived with $100-\gamma=1.0 \%$, (c) scenario $\mathrm{G}$, matrix derived with $100-\gamma=2.0 \%$, and (d) scenario $\mathrm{H}$, matrix derived with $100-\gamma=$ $10.0 \%$.

Figure 4. The real confusion matrices and those derived after the introduction of ground reference data error. (a) the real matrices for maps A and B (based on Stehman (2005), table 2), (b) confusion matrices arising from the introduction of independent errors and (c) confusion matrices arising from the introduction of correlated errors. Note some the analyses required some rounding of numbers and that for the analyses based on map A $S_{1}^{\prime}=S_{2}^{\prime}=95.0 \%$ while with map B $S_{1}^{\prime}=S_{2}^{\prime}=98.0 \%$.

Figure 5. The observed and real confusion matrices together with associated derived values for scenarios I-L.

Figure 6. Variation in perceived sensitivity (dark line) and specificity (dashed line) with prevalence. (a) For $S_{1}^{\prime}=S_{2}^{\prime}=95.0 \%, S_{1}=S_{2}=90.0 \%$; note scenario A had these settings and $\theta=20 \%$, (b) $S_{1}^{\prime}=S_{2}^{\prime}=90.0 \%, S_{1}=S_{2}=80.0 \%$; note scenarios B and $\mathrm{E}$ had these settings and $\theta=20 \%$ and $\theta=5 \%$ respectively, (c) $S_{1}^{\prime}=S_{2}^{\prime}=70.0 \%, S_{1}=S_{2}=65.0 \%$; note scenario $\mathrm{C}$ had these settings and $\theta=20 \%$, and (d) $S_{1}^{\prime}=90.0 \%, S_{2}^{\prime}=100.0 \%, S_{1}=S_{2}=80.0 \%$; note scenario $\mathrm{D}$ had these settings and $\theta=20 \%$. 


\section{Ground reference $\downarrow$}

\section{Remote sensing $\longrightarrow$}

\begin{tabular}{r|c|c|c|}
\multicolumn{4}{c}{ Change } \\
change & $\Sigma$ \\
\cline { 2 - 4 } Change & $a$ & $b$ & $g$ \\
\cline { 2 - 4 } No change & $c$ & $d$ & $h$ \\
\cline { 2 - 4 }$\Sigma$ & $e$ & $f$ & $n$ \\
\cline { 2 - 4 } & & &
\end{tabular}

Figure 1. The binary confusion matrix. Each case is allocated to one of the elements of the $2 \times 2$ matrix (highlighted in grey) and a suite of statistics may be derived from the matrix entries relative to the marginal values. All other matrices presented in this paper are formatted in the style shown here. 
Scenario

A

\begin{tabular}{|r|r|r|}
\hline 175 & 85 & 260 \\
\hline 55 & 685 & 740 \\
\hline 230 & 770 & 1000 \\
\hline
\end{tabular}

$\hat{S}_{1}=76.1 \%$
$\hat{U}_{1}=67.3 \%$
$\hat{\theta}=23.0 \%$

\begin{tabular}{|r|r|r|}
\hline 160 & 160 & 320 \\
\hline 100 & 580 & 680 \\
\hline 260 & 740 & 1000 \\
\hline
\end{tabular}

$\hat{S}_{1}=61.5 \%$

$\hat{U}_{1}=50.0 \%$

$\hat{\theta}=26.0 \%$

\begin{tabular}{|r|r|r|}
\hline 175 & 235 & 410 \\
\hline 205 & 385 & 590 \\
\hline 380 & 620 & 1000 \\
\hline
\end{tabular}

$\hat{S_{1}}=46.1 \%$
$\hat{U}_{1}=42.7 \%$
$\hat{\theta}=38.0 \%$

D

\begin{tabular}{|r|r|r|}
\hline 128 & 192 & 320 \\
\hline 32 & 648 & 680 \\
\hline 160 & 840 & 1000 \\
\hline
\end{tabular}

$\hat{S}_{1}=80.0 \%$

$\hat{U}_{1}=40.0 \%$

$\hat{\theta}^{1}=16.0 \%$

\begin{tabular}{|r|r|r|}
\hline 55 & 175 & 230 \\
\hline 85 & 685 & 770 \\
\hline 140 & 860 & 1000 \\
\hline
\end{tabular}

$\hat{S}_{1}=39.3 \%$
$\hat{U}_{1}=23.9 \%$
$\hat{\theta}=14.0 \%$

\begin{tabular}{|r|r|r|}
\hline 130 & 280 & 410 \\
\hline 70 & 520 & 590 \\
\hline 200 & 800 & 1000 \\
\hline
\end{tabular}

$S_{1}=65.0 \%$

$U_{1}=31.7 \%$

$\theta=20.0 \%$

\begin{tabular}{|r|r|r|}
\hline 180 & 80 & 260 \\
\hline 20 & 720 & 740 \\
\hline 200 & 800 & 1000 \\
\hline
\end{tabular}

$S_{1}=90.0 \%$

$U_{1}=69.2 \%$

$\theta=20.0 \%$

\begin{tabular}{|r|r|r|}
\hline 160 & 160 & 320 \\
\hline 40 & 640 & 680 \\
\hline 200 & 800 & 1000 \\
\hline
\end{tabular}

$S_{1}=80.0 \%$

$U_{1}=50.0 \%$

$\theta=20.0 \%$

\begin{tabular}{|r|r|r|}
\hline 160 & 160 & 320 \\
\hline 40 & 640 & 680 \\
\hline 200 & 800 & 1000 \\
\hline
\end{tabular}

$S_{1}=80.0 \%$

$U_{1}=50.0 \%$

$\theta=20.0 \%$

\begin{tabular}{|r|r|r|}
\hline 40 & 190 & 230 \\
\hline 10 & 760 & 770 \\
\hline 50 & 950 & 1000 \\
\hline
\end{tabular}

$S_{1}=80.0 \%$

$U_{1}=17.4 \%$

$\theta=5.0 \%$

Figure 2.The observed and real confusion matrices based on imperfect and perfect ground reference data respectively together with associated derived values for scenarios A-E. Note that in each of the scenarios depicted the data were generated in a fashion to ensure that the assumption of conditional independence was satisfied. 


\begin{tabular}{|c|c|c|c|}
\hline 160 & 160 & 320 & $S_{1}=80.0 \%$ \\
\hline 40 & 640 & 680 & $U_{1}=50.0 \%$ \\
\hline 200 & 800 & 1000 & $\theta=20.0 \%$ \\
\hline
\end{tabular}

(a)

\begin{tabular}{|r|r|r|}
\hline 176 & 144 & 320 \\
\hline 36 & 644 & 680 \\
\hline 212 & 788 & 1000 \\
\hline
\end{tabular}

(c)

$$
\begin{aligned}
& \hat{S}_{1}=83.0 \% \\
& \hat{U}_{1}=55.0 \% \\
& \hat{\theta}=21.2 \%
\end{aligned}
$$

\begin{tabular}{|r|r|r|}
\hline 168 & 152 & 320 \\
\hline 38 & 642 & 680 \\
\hline 206 & 794 & 1000 \\
\hline
\end{tabular}

$\hat{S_{1}}=81.5 \%$

$\hat{U}_{1}=52.5 \%$

$\hat{\theta}=20.6 \%$

(b)

\begin{tabular}{|r|r|r|}
\hline 240 & 80 & 320 \\
\hline 20 & 660 & 680 \\
\hline 260 & 740 & 1000 \\
\hline
\end{tabular}

$\hat{S}_{1}=92.3 \%$
$\hat{U}_{1}=75.0 \%$
$\hat{\theta}=26.0 \%$

(d)

Figure 3. The confusion matrices and derived values illustrating impacts of correlated errors. (a) the real matrix, (b) scenario F, matrix derived with $100-\gamma=1.0 \%$, (c) scenario $\mathrm{G}$, matrix derived with $100-\gamma=2.0 \%$, and (d) scenario $\mathrm{H}$, matrix derived with $100-\gamma=$ $10.0 \%$. 
Map A

\begin{tabular}{|r|r|r|}
\hline 134 & 49 & 183 \\
\hline 43 & 774 & 817 \\
\hline 177 & 823 & 1000 \\
\hline
\end{tabular}

Map B

\begin{tabular}{|r|r|r|}
\hline 257 & 17 & 274 \\
\hline 24 & 702 & 726 \\
\hline 281 & 719 & 1000 \\
\hline
\end{tabular}

$S_{1}=91.4 \%$

$U_{1}=93.9 \%$

$\theta=28.1 \%$

(a)

\begin{tabular}{|r|r|r|}
\hline 130 & 53 & 183 \\
\hline 79 & 738 & 817 \\
\hline 209 & 791 & 1000 \\
\hline
\end{tabular}

$\hat{S_{1}}=62.2 \%$
$\hat{U_{1}}=71.0 \%$
$\hat{\theta}=20.9 \%$

\begin{tabular}{|r|r|r|}
\hline 252 & 22 & 274 \\
\hline 37 & 689 & 726 \\
\hline 289 & 711 & 1000 \\
\hline
\end{tabular}

$\hat{S}_{1}=87.2 \%$
$\hat{U}_{1}=91.9 \%$
$\hat{\theta}=28.9 \%$

(b)

\begin{tabular}{|r|r|r|}
\hline 175 & 8 & 183 \\
\hline 34 & 783 & 817 \\
\hline 209 & 791 & 1000 \\
\hline
\end{tabular}

$\hat{S_{1}}=83.7 \%$
$\hat{\hat{U}_{1}}=95.6 \%$
$\hat{\theta}=20.9 \%$

\begin{tabular}{|r|r|r|}
\hline 271 & 3 & 274 \\
\hline 18 & 708 & 726 \\
\hline 289 & 711 & 1000 \\
\hline
\end{tabular}

$\hat{S}_{1}=93.7 \%$

$\hat{U}_{1}=98.9 \%$

$\hat{\theta}^{1}=28.9 \%$

(c)

Figure 4. The real confusion matrices and those derived after the introduction of ground reference data error. (a) the real matrices for maps A and B (based on Stehman (2005), table 2), (b) confusion matrices arising from the introduction of independent errors and (c) confusion matrices arising from the introduction of correlated errors. Note some the analyses required some rounding of numbers and that for the analyses based on map A $S_{1}^{\prime}=S_{2}^{\prime}=95.0 \%$ while with map B $S_{1}^{\prime}=S_{2}^{\prime}=98.0 \%$. 
Scenario

\begin{tabular}{|c|c|c|c|}
\hline 174 & 87 & 320 & $\hat{S}_{1}=66.9 \%$ \\
\hline 86 & 653 & 680 & $\hat{U}_{1}=54.3 \%$ \\
\hline 260 & 740 & 1000 & $\lambda$ \\
\hline
\end{tabular}

\begin{tabular}{|r|r|r|}
\hline 163 & 118 & 281 \\
\hline 97 & 622 & 719 \\
\hline 260 & 740 & 1000 \\
\hline
\end{tabular}

\begin{tabular}{|r|r|r|}
\hline 152 & 229 & 381 \\
\hline 108 & 511 & 619 \\
\hline 260 & 740 & 1000 \\
\hline
\end{tabular}

\begin{tabular}{|r|r|r|}
\hline 195 & 124 & 319 \\
\hline 65 & 616 & 681 \\
\hline 260 & 740 & 1000 \\
\hline
\end{tabular}

$\hat{S}_{1}=62.7 \%$

$\hat{U}_{1}=58.0 \%$

$\hat{\theta}=26.0 \%$

$\hat{S}_{1}=58.5 \%$

$\hat{U}_{1}=39.9 \%$

$\hat{\theta}=26.0 \%$

$\hat{S_{1}}=75.0 \%$
$\hat{U_{1}}=61.1 \%$
$\hat{\theta}=14.0 \%$

\begin{tabular}{|r|r|r|}
\hline 165 & 116 & 281 \\
\hline 35 & 684 & 719 \\
\hline 200 & 800 & 1000 \\
\hline
\end{tabular}

$S_{1}=82.5 \%$

$U_{1}=58.7 \%$

$\theta=20.0 \%$

\begin{tabular}{|r|r|r|}
\hline 134 & 247 & 381 \\
\hline 66 & 553 & 619 \\
\hline 200 & 800 & 1000 \\
\hline
\end{tabular}

$S_{1}=67.0 \%$

$U_{1}=35.2 \%$

$\theta=20.0 \%$

\begin{tabular}{|r|r|r|}
\hline 133 & 186 & 319 \\
\hline 67 & 614 & 681 \\
\hline 200 & 800 & 1000 \\
\hline
\end{tabular}

$S_{1}=66.5 \%$

$U_{1}=41.7 \%$

$\theta=20.0 \%$

Figure 5. The observed and real confusion matrices together with associated derived values for scenarios I-L. 


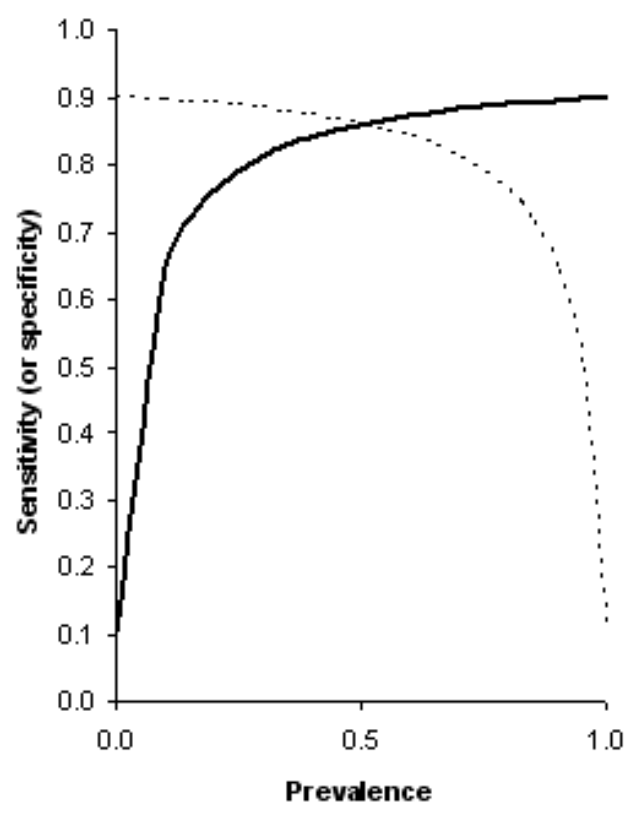

(a)

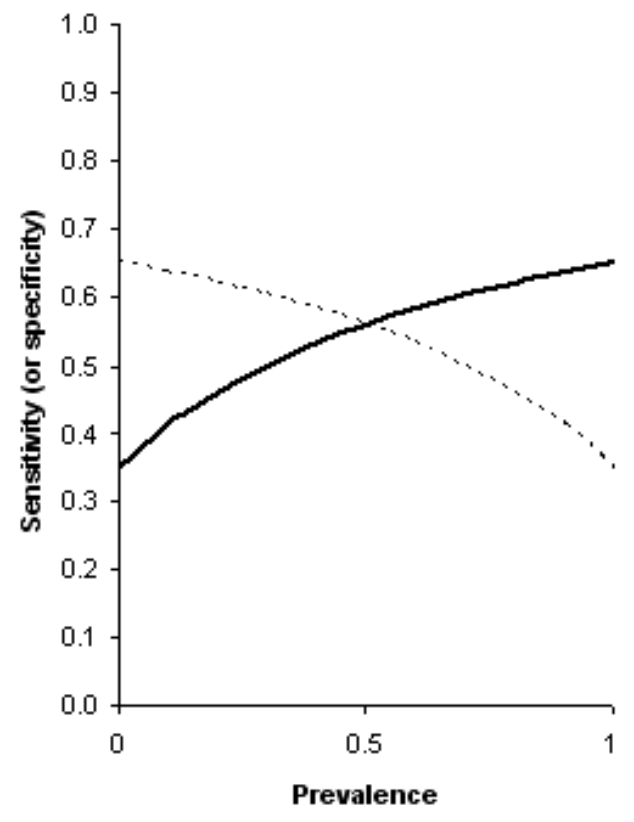

(c)

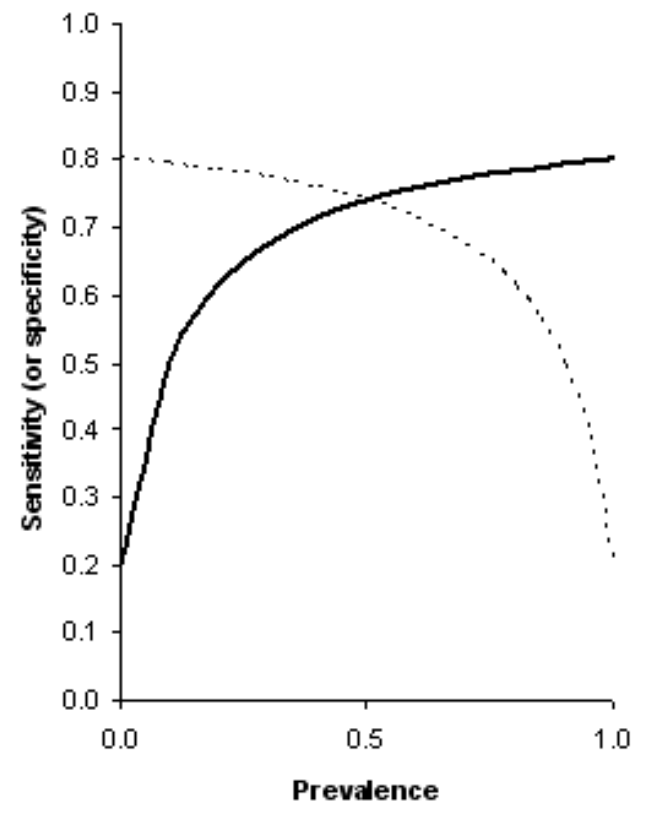

(b)

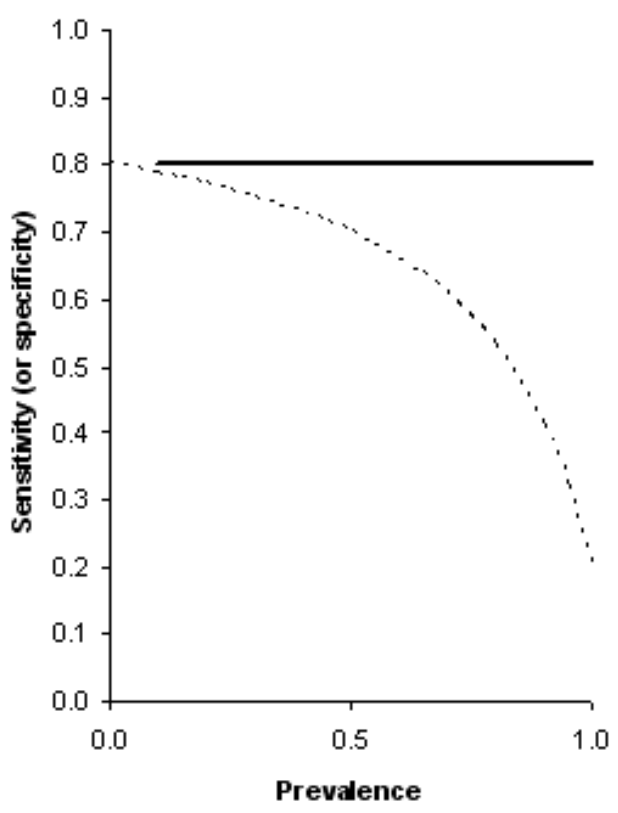

(d)

Figure 6. Variation in observed or perceived sensitivity (dark line) and specificity (dashed line) with prevalence. (a) For $S_{1}^{\prime}=S_{2}^{\prime}=95.0 \%, S_{1}=S_{2}=90.0 \%$; note scenario A had these settings and $\theta=20 \%$, (b) $S_{1}^{\prime}=S_{2}^{\prime}=90.0 \%, S_{1}=S_{2}=80.0 \%$; note scenarios B and $\mathrm{E}$ had these settings and $\theta=20 \%$ and $\theta=5 \%$ respectively, (c) $S_{1}^{\prime}=S_{2}^{\prime}=70.0 \%, S_{1}=S_{2}=65.0 \%$; note scenario $\mathrm{C}$ had these settings and $\theta=20 \%$, and (d) $S_{1}^{\prime}=90.0 \%, S_{2}^{\prime}=100.0 \%$, $S_{1}=S_{2}=80.0 \%$; note scenario D had these settings and $\theta=20 \%$. 